\title{
A REAL-SPACE MODAL ANALYSIS METHOD FOR NON- PROPORTIONAL DAMPED STRUCTURES
}

\author{
E. Stanoev ${ }^{1}$ \\ ${ }^{1}$ Chair of Wind Energy Technology, \\ Faculty of Mechanical Engineering and Marine Technology, \\ University of Rostock, Albert-Einstein-Str. 2, 18059 Rostock, Germany \\ e-mail:evgueni.stanoev@uni-rostock.de
}

Keywords: modal decomposition of the equations of motion, complex eigenvalue problem, proportional/non-proportional damping, synchronous free vibrations

\begin{abstract}
The inclusion of damping in the equations of motion of FEM-based structural models yields a complex (quadratic) eigenvalue problem. In this paper is presented a variant of a general method [4], [5] for real-space modal transformation of damped multi-degree-offreedom-systems (MDOFS) with non-modal (non-proportional) symmetric damping matrix. The method is based on the conjugated complex right eigenvectors of the system, normalized relative to the general mass matrix. After state-space formulation of the equations of motion a real modal transformation matrix is built by a combination of two complex transformations, which is the main advantage of the presented method. Analytically expressions for the modal transformation basis are developed be the aid of computer algebra software (MATLAB).
\end{abstract}

Applying the suggested method to the special case of proportionally damped system, an analytical expression for the constant phase lag of the free vibration modes has been derived. The conversion of the developed general real transformation matrix into the modal matrix of the undamped problem is analytically proved by taking into account the synchronous free oscillations in this special case.

The derived formulas for the modal transformation basis contain the real and the imaginary parts of the eigenvectors and the associated eigenvalues.

A numerical example - vibration of a rotor blade of a wind turbine - demonstrates the performance of the presented modal decomposition method for the general case of nonproportional damped system. The damping matrix of this example contains structural and aerodynamic damping. The initial computation of the complex eigensolution of the FEM beam model in the presented example and all subsequent computations are done by the aid of the Symbolic Math Toolbox of MATLAB. The suggested procedure can be applied in structural systems containing different damping and energy-loss mechanism in various parts of the structure and also in structure-environment interaction problems, where a non-modal damping matrix is occurring. 


\section{INTRODUCTION}

The modal decomposition of the equations of motion of multi-degree-of-freedom-systems (MDOFS) is usually applied to systems without damping. The associated eigenvalue problem has real eigenvectors and real free frequencies. The inclusion of damping in the equations of MDOFS leads to a quadratic eigenvalue problem with complex conjugate pairs of eigenvalues and eigenmodes. The modal decomposition of the equations has to be performed in complex space. Aiming to avoid the computation in complex arithmetic, a new modal decomposition method, presented in details in [2] - [5], is briefly outlined in Sec. 2. This procedure is based on a real modal transformation matrix, derived from the complex eigenvalue solution of a MDOFS with symmetric non-proportional (non-modal) damping matrix.

In the suggested procedure the complex eigenvectors and eigenvalues of the structural model should be computed first. In the presented example in Sec. 4 - vibration of a rotor blade of a wind generator - computer algebra software was applied to solve the eigenvalue problem. In real life applications of the presented method to high dimensional problems it must be available a reliable eigenmode solver for large complex eigenvalue computations. There are many literature references for large scaled problems with various solution strategies, see [10] - [12]. The author has used an implicitly restarted Arnoldi/Lanczos method [11], [12] to solve the complex eigenvalue problem in an application of the method to a fluid-structurefoundation interaction problem, see in [1],[2].

Another topic of this paper is to show an analytical proof of the statement for the constant phase lag/lead of free vibrations in the proportional damping case - see the introduction in Sec. 1.2. The analytical proof in an indirect manner is based on the procedure, summarized in Sec. 2. A formula for computing of the constant ratio $\frac{\operatorname{Im}(\mathbf{X})_{k}}{\operatorname{Re}(\mathbf{X})_{k}}$ has been derived in Sec. 3.1.

In Sec. 4 the proposed modal analysis method, presented in Section 2, has been applied to a rotor blade beam structure with 54 DOF. The numerical example demonstrates the performance of the method for the general case of non-proportional damping. In this case the damping matrix of the system contains a stiffness proportional (Rayleigh) damping and aerodynamic (non-proportional) damping parts. In the second variant of the solution - with proportional damping matrix, the formula for the constant phase of the resonance modes is verified numerically.

\subsection{Free vibrations of a viscously damped system}

The equations of motion of a damped MDOFS are

$$
\mathbf{M} \ddot{\mathbf{V}}+\mathbf{D} \dot{\mathbf{V}}+\mathbf{K V}=\mathbf{p}(t)
$$

where $\mathbf{M}, \mathbf{D}$ and $\mathbf{K}$ are, respectively the ( $\mathrm{n} \times \mathrm{n}$ ) mass, damping and stiffness matrices, and $\mathbf{V}$, $\dot{\mathbf{V}}$ are the ( $\mathrm{n} \times 1$ ) displacement and velocity vectors and $\mathbf{p}(\mathrm{t})$ is the ( $\mathrm{n} \times 1)$ excitation vector.

In structural mechanics problems we consider the $\mathbf{M}$ and $\mathbf{K}$ matrices to be real, symmetric and positive definite, excluding the presence of rigid body modes. The $\mathbf{D}$ matrix is assumed to be symmetric, non-negative, she presents a non-proportional damping. 
With the assumed free vibration in the form

$\mathbf{V}=\mathbf{X} e^{\lambda t}, \quad \dot{\mathbf{V}}=\lambda \mathbf{X} e^{\lambda t}$,

the associated quadratic eigenvalue problem is

$\left(\lambda_{j}^{2} \mathbf{M}+\lambda_{j} \mathbf{D}+\mathbf{K}\right) \mathbf{X}_{j}=\mathbf{0} \quad \forall(j=1, \cdots, n)$

In Eq. (1.3) the $\mathrm{j}^{\text {th }}$ eigenvalue $\lambda_{j}$ and the corresponding eigenmode $\mathbf{X}_{j}$ appear in complex conjugate pairs (index j omitted):

$\lambda=\lambda_{r}+i \lambda_{i}, \quad \bar{\lambda}=\lambda_{r}-i \lambda_{i}$

$\mathbf{X}=\mathbf{X}_{r}+i \mathbf{X}_{i}, \quad \overline{\mathbf{X}}=\mathbf{X}_{r}-i \mathbf{X}_{i}$

The dynamic equilibrium of a viscously damped single oscillator is governed by

$m \ddot{v}(t)+c \dot{v}(t)+k v(t)=q(t) \quad$ resp.

$\ddot{v}(t)+2 \eta \omega \dot{v}(t)+\omega^{2} v(t)=p(t)$

where $\quad \ddot{v} \quad$ is acceleration,

$\dot{v} \quad$ - velocity,

$\omega=\sqrt{\frac{k}{m}} \quad$ - free vibration frequency,

$\eta=\frac{c}{2 m \omega} \quad$ - Lehr's damping ratio and $\quad p(t)=\frac{q(t)}{m}$.

The exponential solution $x e^{\lambda t}$, introduced into the homogenous form of the differential equation (1.5b), yields the eigenvalue problem

$\lambda^{2}+2 \eta \omega \lambda+\omega^{2}=0$

The eigenvalue solution (assuming that $\eta \ll 1$, subcritical damping) of Eq. (1.6) is a complex conjugate pair:

$\lambda_{1 / 2}=\underbrace{-\eta \omega}_{\lambda_{r}} \pm i \underbrace{\omega \sqrt{1-\eta^{2}}}_{\lambda_{i}=\omega_{D}}=\lambda_{r} \pm i \lambda_{i}$

\subsection{The constant phase lag problem}

Interpreting the eigenvalue pair (1.4a) as the single-oscillator-eigenvalues (1.7), we can express the $\mathrm{j}^{\text {th }}$ free vibration of the MDOFS as linear combination of the two complex conjugate eigenpairs $(1.4 \mathrm{a}, \mathrm{b})$ :

$\mathbf{V}=\mathbf{X} e^{\lambda t}=\mathbf{X} e^{\left(-\eta \omega \pm i \omega \sqrt{1-\eta^{2}}\right) t}=$

$=e^{-\eta \omega t}\left[\left(\mathbf{X}_{r}+i \mathbf{X}_{i}\right)\left(\cos \omega_{D} t+i \sin \omega_{D} t\right)+\left(\mathbf{X}_{r}-i \mathbf{X}_{i}\right)\left(\cos \omega_{D} t-i \sin \omega_{D} t\right)\right]$

$=e^{-\eta \omega t}\left[\begin{array}{c}2\left(\mathbf{X}_{r} \cos \omega_{D} t-\mathbf{X}_{i} \sin \omega_{D} t\right)+ \\ i\left(\mathbf{X}_{i} \cos \omega_{D} t+\mathbf{X}_{r} \sin \omega_{D} t\right)-i\left(\mathbf{X}_{i} \cos \omega_{D} t+\mathbf{X}_{r} \sin \omega_{D} t\right)\end{array}\right]$ 
$=e^{-\eta \omega t}[\underbrace{2 \mathbf{X}_{r}}_{\mathbf{F} \cos \varphi} \cos \omega_{D} t-\underbrace{2 \mathbf{X}_{i}}_{\mathbf{F} \sin \varphi} \sin \omega_{D} t]$

The last relation leads to the real form of a damped free oscillation for every $\mathrm{k}^{\text {th }} \mathrm{DOF}$ :

$V_{k}=e^{-\eta \omega t}\left[F_{k} \cos \left(\omega_{D} t+\varphi_{k}\right)\right]$

where $\quad \varphi_{k}=\arctan \frac{\left(X_{i}\right)_{k}}{\left(X_{r}\right)_{k}} \quad:$ phase lag/lead for the $\mathrm{k}^{\text {th }} \mathrm{DOF}$

Since the viscous damping is assumed to be non-proportional, the free vibration solution (1.9) represents non-synchronous damped oscillation (i.e. the phase $\varphi_{k}$ is different for each DOF). In the case of proportionally damped system we have to deal with synchronous free oscillation - i.e. the phase $\varphi_{k}$ is constant (the same for all DOF), for undamped systems $\varphi_{k}$ is zero - see [6], [7], p.118.

The features, showed in Eq.(1.9), (1.10) are well known and used in modal analysis, see for example [6]. In the present paper the statement of synchronous free oscillations in the proportional damping case should be proved analytically in Sec. 3.1.

\section{MODAL DECOMPOSITION METHOD INCLUDING THE COMPLEX RIGHT EIGENVECTORS}

\subsection{The single mass oscillator}

The equation of motion of a damped single degree of freedom system (SDOFS) (1.5b) can be written in the form

$$
\begin{aligned}
& \underbrace{\left[\begin{array}{rr}
1 & 0 \\
0 & -\omega^{2}
\end{array}\right]}_{\mathbf{m}} \underbrace{\left[\begin{array}{c}
\dot{w}(t) \\
\dot{v}(t)
\end{array}\right]}_{\mathbf{q}}+\underbrace{\left[\begin{array}{cc}
2 \eta \omega & \omega^{2} \\
\omega^{2} & 0
\end{array}\right]}_{\mathbf{k}} \underbrace{\left[\begin{array}{c}
w(t) \\
v(t)
\end{array}\right]}_{\mathbf{q}}=\underbrace{\left[\begin{array}{c}
p(t) \\
0
\end{array}\right]}_{\mathbf{p}(t)} \\
& \mathbf{m} \dot{\mathbf{q}}+\mathbf{k} \mathbf{q}=\mathbf{p} \quad \rightarrow \quad \dot{\mathbf{q}}+\underbrace{\left(\mathbf{m}^{-1} \mathbf{k}\right)}_{\underline{\mathbf{a}}} \mathbf{q}=\mathbf{m}^{-1} \mathbf{p}
\end{aligned}
$$

where the velocity is

$$
w=\dot{v}
$$

The exponential solution $\mathbf{q}=\mathbf{x} e^{\lambda t}, \quad \dot{\mathbf{q}}=\lambda \mathbf{x} e^{\lambda t}$, introduced into the homogenous form of the differential equation (2.1), gives the quadratic eigenvalue problem

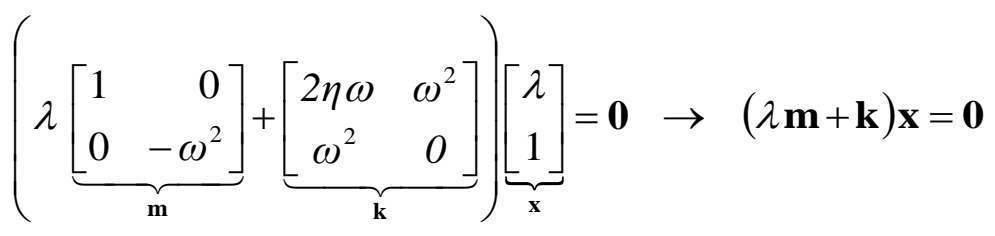

The two complex conjugate eigenvalues ( $\eta<<1$, subcritical damped system) are: 
$\lambda_{1 / 2}=-\eta \omega \pm \sqrt{(\eta \omega)^{2}-\omega^{2}}=\underbrace{-\eta \omega}_{\lambda_{r}} \pm i \underbrace{\omega \sqrt{1-\eta^{2}}}_{\lambda_{i}}=\lambda_{r} \pm i \lambda_{i}$

where $\quad \omega=\sqrt{\left(\lambda_{r}\right)^{2}+\left(\lambda_{i}\right)^{2}}, \quad \eta=-\frac{\lambda_{r}}{\omega}, \quad \omega_{D}=\omega \sqrt{1-\eta^{2}}$

The two corresponding complex conjugate eigenvectors $\varphi_{1 / 2}$, at first normalized relative to the mass matrix

$$
\varphi_{\mathbf{k}}=\frac{\mathbf{x}_{\mathbf{k}}}{\sqrt{\mathbf{x}_{\mathbf{k}}^{\mathbf{T}} \mathbf{m} \mathbf{x}_{\mathbf{k}}}}=\frac{1}{\sqrt{-\omega^{2}+\left(-\lambda_{r} \pm i \lambda_{i}\right)^{2}}}\left[\begin{array}{c}
\lambda_{r} \pm i \lambda_{i} \\
1
\end{array}\right], \quad(k=1,2)
$$

are combined into a modal matrix:

$$
\varphi=\left[\begin{array}{ll}
\varphi_{1} & \varphi_{2}
\end{array}\right]
$$

Due to normalization Eq. (2.6) the orthogonality relationships can be derived:

$$
\begin{aligned}
& \varphi^{\mathbf{T}} \mathbf{m} \varphi=\varphi^{\mathbf{T}}\left[\begin{array}{cc}
1 & 0 \\
0 & -\omega^{2}
\end{array}\right] \varphi=\left[\begin{array}{ll}
1 & 0 \\
0 & 1
\end{array}\right] \leftrightarrow \varphi^{-\mathbf{T}}\left[\begin{array}{cc}
1 & 0 \\
0 & 1
\end{array}\right] \varphi^{-\mathbf{1}}=\left[\begin{array}{cc}
1 & 0 \\
0 & -\omega^{2}
\end{array}\right] \\
& \varphi^{\mathbf{T}} \mathbf{k} \varphi=\varphi^{\mathbf{T}}\left[\begin{array}{cc}
2 \eta \omega & \omega^{2} \\
\omega^{2} & 0
\end{array}\right] \varphi=\left[\begin{array}{cc}
-\lambda_{1} & 0 \\
0 & -\lambda_{2}
\end{array}\right] \leftrightarrow \varphi^{-\mathbf{T}}\left[\begin{array}{cc}
-\lambda_{1} & 0 \\
0 & -\lambda_{2}
\end{array}\right] \varphi^{-\mathbf{1}}=\left[\begin{array}{cc}
2 \eta \omega & \omega^{2} \\
\omega^{2} & 0
\end{array}\right]
\end{aligned}
$$

The inverse of the complex modal matrix $\varphi(\omega, \eta)$ can be expressed analytically using computer algebra software:

$$
\varphi^{-1}=\frac{1}{2 \sqrt{1-\eta^{2}}}\left[\begin{array}{cc}
\left(-Z_{1}-i Z_{2}\right) & P-i Q \\
\left(-Z_{1}+i Z_{2}\right) & P+i Q
\end{array}\right]
$$

where

$$
\begin{array}{ll}
Z_{1}=\sqrt{\sqrt{1-\eta^{2}}+\left(1-\eta^{2}\right)} \quad Z_{2}=\sqrt{\sqrt{1-\eta^{2}}-\left(1-\eta^{2}\right)} \\
P=\omega\left(\sqrt{1-\eta^{2}} Z_{2}-\eta Z_{1}\right) & Q=\omega\left(\sqrt{1-\eta^{2}} Z_{1}+\eta Z_{2}\right)
\end{array}
$$

\subsection{The damped multi-degree-of-freedom-system}

The equations of motion (1.1) of damped MDOFS (n DOF) will be written in the statespace form:

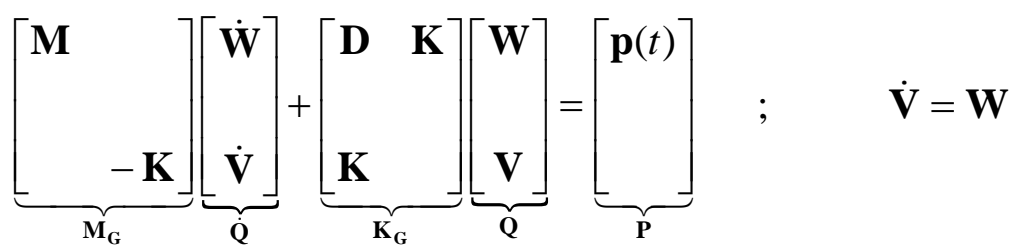

$\mathbf{M}_{\mathbf{G}} \dot{\mathbf{Q}}+\mathbf{K}_{\mathbf{G}} \mathbf{Q}=\mathbf{P}$, 
where $\mathbf{M}_{\mathbf{G}}$ and $\mathbf{K}_{\mathbf{G}}$ are, respectively the ( $\left.2 n \times 2 n\right)$ symmetric generalized mass and the generalized stiffness matrices. The symmetric damping matrix $\mathbf{D}$ is non-negative and represents a non-proportional damping.

The exponential solution (1.2), substituted into the homogenous form of Eq.(2.12), leads to the $2 n$-dimensional eigenvalue problem

$\left(\lambda \mathbf{M}_{\mathbf{G}}+\mathbf{K}_{\mathbf{G}}\right)\left[\begin{array}{c}\lambda \mathbf{X} \\ \mathbf{X}\end{array}\right]=\mathbf{0}$

The solution of Eq. (2.13) is given by n complex conjugate eigenpairs (1.4), now written in the form:

$\lambda^{(j)}=\lambda_{r}^{(j)}+i \lambda_{i}^{(j)} \quad \rightarrow\left[\begin{array}{c}\lambda^{(j)} \mathbf{X}^{(j)} \\ \mathbf{X}^{(j)}\end{array}\right] ; \quad \bar{\lambda}^{(j)}=\lambda_{r}^{(j)}-i \lambda_{i}^{(j)} \quad \rightarrow\left[\begin{array}{c}\bar{\lambda}^{(j)} \overline{\mathbf{X}}^{(j)} \\ \overline{\mathbf{X}}^{(j)}\end{array}\right] ; \quad(j=1,2 \ldots n)$

Each $\mathrm{j}^{\text {th }}$ eigenvector-pair $\mathbf{X}^{(j)}, \overline{\mathbf{X}}^{(j)}$ is normalized (index (j) omitted) relative to the general mass matrix $\mathbf{M}_{\mathbf{G}}$ :

$$
\begin{array}{ll}
\mathbf{\Phi}=\frac{\mathbf{X}}{\sqrt{A+i B}}=\boldsymbol{\Phi}_{\mathbf{r}}+i \boldsymbol{\Phi}_{\mathbf{i}}, & A+i B=\left[\begin{array}{c}
\lambda \mathbf{X} \\
\mathbf{X}
\end{array}\right]^{T} \mathbf{M}_{\mathbf{G}}\left[\begin{array}{c}
\lambda \mathbf{X} \\
\mathbf{X}
\end{array}\right] \\
\overline{\mathbf{\Phi}}=\frac{\overline{\mathbf{X}}}{\sqrt{A-i B}}=\boldsymbol{\Phi}_{\mathbf{r}}-i \boldsymbol{\Phi}_{\mathbf{i}}, & A-i B=\left[\begin{array}{c}
\bar{\lambda} \overline{\mathbf{X}} \\
\overline{\mathbf{X}}
\end{array}\right]^{T} \mathbf{M}_{\mathbf{G}}\left[\begin{array}{c}
\bar{\lambda} \overline{\mathbf{X}} \\
\overline{\mathbf{X}}
\end{array}\right]
\end{array}
$$

Subject to the normalization (2.15) follow the orthogonality relationships -expressed in terms of the $\mathrm{j}^{\text {th }}$ eigenvector-pair (index (j) omitted):

$$
\begin{aligned}
& {\left[\begin{array}{cc}
\lambda \boldsymbol{\Phi} & \bar{\lambda} \overline{\boldsymbol{\Phi}} \\
\mathbf{\Phi} & \overline{\boldsymbol{\Phi}}
\end{array}\right]^{T} \underbrace{\left[\begin{array}{cc}
\mathbf{M} & \\
& -\mathbf{K}
\end{array}\right]}_{\mathbf{M}_{\mathbf{G}}}\left[\begin{array}{cc}
\lambda \boldsymbol{\Phi} & \bar{\lambda} \overline{\boldsymbol{\Phi}} \\
\boldsymbol{\Phi} & \overline{\boldsymbol{\Phi}}
\end{array}\right]=\left[\begin{array}{ll}
1 & \\
& 1
\end{array}\right]} \\
& {\left[\begin{array}{cc}
\lambda \boldsymbol{\Phi} & \bar{\lambda} \overline{\boldsymbol{\Phi}} \\
\boldsymbol{\Phi} & \overline{\boldsymbol{\Phi}}
\end{array}\right]^{T} \underbrace{\left[\begin{array}{cc}
\mathbf{D} & \mathbf{K} \\
\mathbf{K}
\end{array}\right]}_{\mathbf{K}_{\mathbf{G}}}\left[\begin{array}{cc}
\lambda \boldsymbol{\Phi} & \bar{\lambda} \overline{\boldsymbol{\Phi}} \\
\boldsymbol{\Phi} & \overline{\boldsymbol{\Phi}}
\end{array}\right]=\left[\begin{array}{cc}
-\lambda & \\
& -\bar{\lambda}
\end{array}\right]}
\end{aligned}
$$

The (2n x 2n) complex square modal matrix, denoted by $\boldsymbol{\Phi}_{\mathbf{G}}$, is made up of the $\mathrm{n}$ eigenvector-pairs, see Eqn.(2.15):

$$
\boldsymbol{\Phi}_{\mathbf{G}}=\left[\begin{array}{cccccc}
\lambda^{(1)} \boldsymbol{\Phi}^{(1)} & \bar{\lambda}^{(1)} \overline{\boldsymbol{\Phi}}^{(1)} & \ldots & \ldots & \lambda^{(n)} \boldsymbol{\Phi}^{(n)} & \bar{\lambda}^{(n)} \overline{\boldsymbol{\Phi}}^{(n)} \\
\boldsymbol{\Phi}^{(1)} & \overline{\boldsymbol{\Phi}}^{(1)} & \ldots & \ldots & \boldsymbol{\Phi}^{(n)} & \overline{\boldsymbol{\Phi}}^{(n)}
\end{array}\right]
$$

The orthogonality properties - see Eq. (2.16), (2.17), are used to perform a modal decomposition of the equations of motion (2.12):

$$
\underbrace{\boldsymbol{\Phi}_{G}^{T}\left[\begin{array}{rr}
\mathbf{M} & -\mathbf{K}
\end{array}\right] \boldsymbol{\Phi}_{\mathbf{G}}}_{\mathbf{E}} \dot{\mathbf{A}}+\underbrace{\boldsymbol{\Phi}_{G}^{T}\left[\begin{array}{ll}
\mathbf{D} & \mathbf{K} \\
\mathbf{K} &
\end{array}\right] \mathbf{\Phi}_{\mathbf{G}}}_{\operatorname{diag}\left\{-\lambda^{(j)}\right\}} \mathbf{A}=\boldsymbol{\Phi}_{G}^{T}[\mathbf{p}(t)]
$$

where 
$\left[\begin{array}{l}\mathbf{W} \\ \mathbf{V}\end{array}\right]=\boldsymbol{\Phi}_{\mathbf{G}} \cdot \mathbf{A}=\boldsymbol{\Phi}_{\mathbf{G}} \cdot\left[\begin{array}{llll}a^{(1)} & b^{(1)} & \cdots & a^{(n)} b^{(n)}\end{array}\right]^{T}$

is a coordinate transformation, and $a^{(j)}, b^{(j)}$ are new complex variables.

Introducing real modal coordinates $x^{(j)}, y^{(j)}$ for each $\mathrm{j}^{\text {th }}$ eigenpair, i.e.:

$\left[\begin{array}{l}a^{(j)} \\ b^{(j)}\end{array}\right]=\left(\varphi^{(j)}\right)^{-1}\left[\begin{array}{l}x^{(j)} \\ y^{(j)}\end{array}\right]$,

the differential equations (2.19) can be transformed in pairs into the real form of SDOFSequation (index (j) omitted), regarding Eqs.(2.8), (2.9) and using (2.5):

$$
\begin{aligned}
& \underbrace{\varphi^{-\mathbf{T}}\left[\begin{array}{ll}
1 & 0 \\
0 & 1
\end{array}\right] \varphi^{-\mathbf{1}}} \cdot\left[\begin{array}{l}
\dot{x} \\
\dot{y}
\end{array}\right]+\underbrace{\varphi^{-\mathbf{T}}\left[\begin{array}{cc}
-\lambda & 0 \\
0 & -\bar{\lambda}
\end{array}\right] \varphi^{-\mathbf{1}}} \cdot\left[\begin{array}{l}
x \\
y
\end{array}\right]=\underbrace{\varphi^{-\mathbf{T}}\left[\begin{array}{c}
\lambda \mathbf{\Phi}^{T} \mathbf{p}(t) \\
\bar{\lambda} \overline{\mathbf{\Phi}}^{T} \mathbf{p}(t)
\end{array}\right]} \\
& {\left[\begin{array}{cc}
1 & 0 \\
0 & -\omega^{2}
\end{array}\right] \cdot\left[\begin{array}{l}
\dot{x} \\
\dot{y}
\end{array}\right]+\left[\begin{array}{cc}
2 \eta \omega & \omega^{2} \\
\omega^{2} &
\end{array}\right] \cdot\left[\begin{array}{l}
x \\
y
\end{array}\right]=\left[\begin{array}{l}
g(t) \\
h(t)
\end{array}\right]}
\end{aligned}
$$

For each eigenvalue pair $\lambda^{(j)}=\lambda_{r}^{(j)} \pm i \lambda_{i}^{(j)}$ the matrix $[\varphi(\omega, \eta)]^{-1}$ of the corresponding SDOFS can be computed by Eqs. (2.4),(2.5),(2.10), (2.11).

\subsection{The real modal transformation basis}

Using both transformations (2.19) and (2.22), the equations of motion (2.12) will be uncoupled into $\mathrm{n}$ real SDOFS block equations as follows:

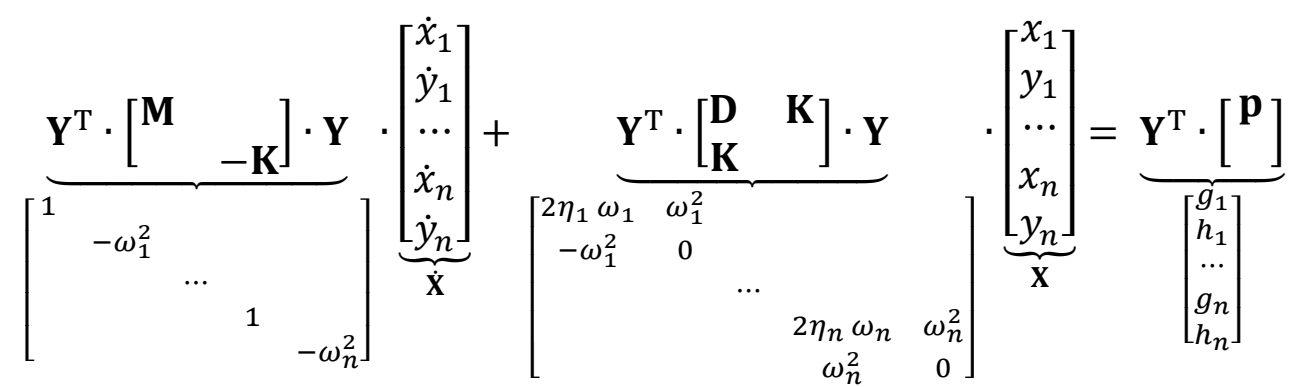

The new $(2 \mathrm{n} \times 2 \mathrm{n})$ transformation basis $\mathbf{Y}$ is defined by combination of two complex transformations (2.19), (2.22):

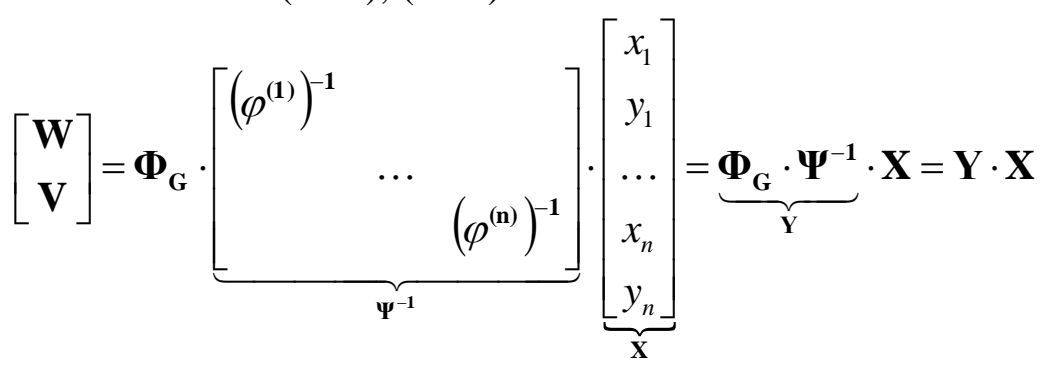

It can be shown that the $\mathbf{Y}$-matrix in Eq. (2.24) and all „load"-vectors $\left[\begin{array}{ll}g(t) & h(t)\end{array}\right]^{T}$, see Eq.(2.22), are purely real. After component multiplication of the analytically expressed terms 
of $\boldsymbol{\Phi}_{G}$ and of $\boldsymbol{\Psi}^{-1}$ all imaginary parts cancel each other, see details in [4]. This is briefly sketched below by developing the two columns of $\mathbf{Y}$, belonging to the $\mathrm{j}^{\text {th }}$ eigenvector-pair:

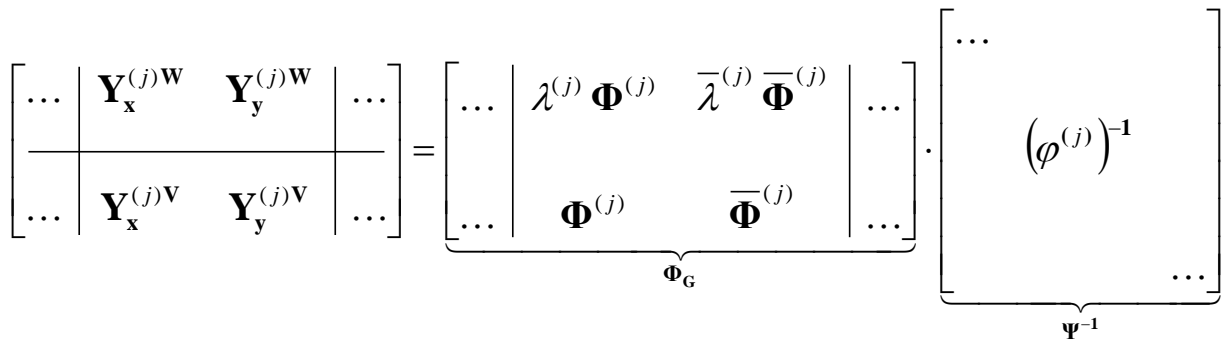

With regard to Eq. (2.5), (2.10), (2.11) the multiplication

$$
\left[\begin{array}{cc}
\left(\lambda_{r}+i \lambda_{i}\right)\left(\boldsymbol{\Phi}_{\mathbf{r}}+i \boldsymbol{\Phi}_{\mathbf{i}}\right) & \left(\lambda_{r}-i \lambda_{i}\right)\left(\boldsymbol{\Phi}_{\mathbf{r}}-i \boldsymbol{\Phi}_{\mathbf{i}}\right) \\
\boldsymbol{\Phi}_{\mathbf{r}}+i \boldsymbol{\Phi}_{\mathbf{i}} & \boldsymbol{\Phi}_{\mathbf{r}}-i \boldsymbol{\Phi}_{\mathbf{i}}
\end{array}\right] \cdot \underbrace{\frac{1}{2 \sqrt{1-\eta^{2}}}\left[\begin{array}{cc}
\left(-Z_{1}-i Z_{2}\right) & P-i Q \\
\left(-Z_{1}+i Z_{2}\right) & P+i Q
\end{array}\right]}_{\left(\varphi^{(j)}\right)^{-1}}
$$

leads to purely real components of the two columns of the transformation basis $\mathbf{Y}$ :

$$
\begin{array}{lcc}
\mathbf{Y}_{\mathbf{x}}^{\mathbf{w}}= & \frac{1}{\sqrt{1-\eta^{2}}}\left\{\left(\omega_{D} Z_{2}+\eta \omega Z_{1}\right) \boldsymbol{\Phi}_{\mathbf{r}}+\left(\omega_{D} Z_{1}-\eta \omega Z_{2}\right) \boldsymbol{\Phi}_{\mathbf{i}}\right\} \\
\mathbf{Y}_{\mathbf{y}}^{\mathbf{w}}= & \frac{1}{\sqrt{1-\eta^{2}}}\left(\omega^{2} Z_{1} \boldsymbol{\Phi}_{\mathbf{r}}-\omega^{2} Z_{2} \mathbf{\Phi}_{\mathbf{i}}\right) \\
\mathbf{Y}_{\mathbf{x}}^{\mathbf{v}}= & \frac{1}{\sqrt{1-\eta^{2}}}\left(-Z_{1} \mathbf{\Phi}_{\mathbf{r}}+Z_{2} \mathbf{\Phi}_{\mathbf{i}}\right) \\
\mathbf{Y}_{\mathbf{y}}^{\mathbf{v}}= & \frac{1}{\sqrt{1-\eta^{2}}}\left\{\left(\omega_{D} Z_{2}-\eta \omega Z_{1}\right) \mathbf{\Phi}_{\mathbf{r}}+\left(\omega_{D} Z_{1}+\eta \omega Z_{2}\right) \mathbf{\Phi}_{\mathbf{i}}\right\}
\end{array}
$$

In the same manner we develop the "load"-vector in Eq. (2.22)

$$
\begin{aligned}
& {\left[\begin{array}{l}
g(t) \\
h(t)
\end{array}\right]=\frac{1}{2 \sqrt{1-\eta^{2}}}\left[\begin{array}{cc}
\left(-Z_{1}-i Z_{2}\right) & \left(-Z_{1}+i Z_{2}\right) \\
P-i Q & P+i Q
\end{array}\right]\left[\begin{array}{l}
\left(\lambda_{r}+i \lambda_{i}\right)\left(\boldsymbol{\Phi}_{\mathbf{r}}^{\mathbf{T}}+i \boldsymbol{\Phi}_{\mathbf{i}}^{\mathbf{T}}\right) \\
\left(\lambda_{r}-i \lambda_{i}\right)\left(\boldsymbol{\Phi}_{\mathbf{r}}^{\mathbf{T}}-i \boldsymbol{\Phi}_{\mathbf{i}}^{\mathbf{T}}\right)
\end{array}\right] \mathbf{p}(t)} \\
& g(t)=\frac{\omega}{\sqrt{1-\eta^{2}}}\left\{\left(Z_{2} \sqrt{1-\eta^{2}}+Z_{1} \eta\right) \boldsymbol{\Phi}_{\mathbf{r}}^{\mathbf{T}}+\left(Z_{1} \sqrt{1-\eta^{2}}-Z_{2} \eta\right) \boldsymbol{\Phi}_{\mathbf{i}}^{\mathbf{T}}\right\} \mathbf{p}(t) \\
& h(t)=\frac{\omega^{2}}{\sqrt{1-\eta^{2}}}\left\{Z_{1} \boldsymbol{\Phi}_{\mathbf{r}}^{\mathbf{T}}-Z_{2} \boldsymbol{\Phi}_{\mathbf{i}}^{\mathbf{T}}\right\} \mathbf{p}(t)
\end{aligned}
$$

Each $\mathrm{j}^{\text {th }}$ SDOFS block equation in (2.23) can be easily solved, eliminating the modal coordinate $x^{(j)}$ to obtain the usual form of the SDOFS equation of motion (index (j) omitted):

$$
\begin{aligned}
& x=\dot{y}+\frac{1}{\omega^{2}} h(t) \\
& \ddot{y}+2 \eta \omega \dot{y}+\omega^{2} y=g(t)-\frac{2 \eta}{\omega} h(t)-\frac{1}{\omega^{2}} \dot{h}(t)
\end{aligned}
$$


The dynamic response $y^{(j)}(t)$ can be obtained by step-by-step integration, applied to Eqs. $(2.30 \mathrm{~b}, \mathrm{a})$. The final time response of the original $\mathrm{n}$ DOFs is calculated by superposition of the modal coordinates $x^{(j)}, y^{(j)}$ in accordance to Eq. (2.24).

The major advantage of the suggested method is the developed new modal transformation matrix Y , see (2.24), in real space for damped MDOFS with symmetric non-diagonalisable (i.e. non-modal) damping matrix. The method has also the usual modal superposition advantage - an uncompleted transformation employing only a few modes $(\mathrm{k}<<\mathrm{n})$ in the $\mathbf{Y}$-basis leads with sufficient numerical accuracy - after the final back coordinate transformation - to the dynamic response of all $\mathrm{n}$ DOF.

\section{THE PROPORTIONAL DAMPED SYSTEM}

\subsection{Modal transformation of the equations of motion}

A simple method to construct a damping matrix $\mathbf{D}_{p}$, presenting a proportional damping, is the Rayleigh damping assumption:

$\mathbf{D}_{\mathbf{p}}=(\alpha \mathbf{M}+\beta \mathbf{K})$

where $\alpha, \beta$ : unknown weighting parameter, see Eq.(3.7),(3.8a,b)

The modal damping matrix is a particular case of a more general proportional damping assumption, see [7] p.105, in the form:

$\mathbf{D}_{\mathbf{p}}=\sum_{k=1}^{n} a_{k} \mathbf{M}\left(\mathbf{M}^{-1} \mathbf{K}\right)^{k-1}$

The matrix (3.1b) turns for $n=2$ to $\mathbf{D}_{\mathbf{p}}=a_{1} \mathbf{M}+a_{2} \mathbf{K}$, which is the Rayleigh approach (3.1a).

The eigenvalue problem

$\left(\lambda_{j}^{2} \mathbf{M}+\mathbf{K}\right) \mathbf{X}_{j}=\mathbf{0}$

corresponding to the equations of motion of MDOFS without damping

$\mathbf{M} \ddot{\mathbf{V}}+\mathbf{K V}=\mathbf{p}(t)$

has the solution: $\quad \lambda_{j}=i \omega_{0 j} \quad$ with the free frequency $\omega_{0 j}$

$\mathbf{X}_{j},(j=1,2 \cdots, n)$ real eigenvectors

The modal matrix $\mathbf{U}$, belonging to (3.2)

$\mathbf{U}=\left[\begin{array}{llll}\mathbf{U}_{1} & \mathbf{U}_{2} & \cdots & \mathbf{U}_{n}\end{array}\right]$

comprises $\mathrm{n}$ real, mass normalized eigenvectors

$\mathbf{U}_{j}=\frac{\mathbf{x}_{j}}{\sqrt{\mathbf{X}_{j}^{T} \mathbf{M} \mathbf{X}_{j}}} \quad(j=1,2, \cdots, n)$. 
The mass normalisation (3.5) leads to the orthogonality relationships

$\begin{aligned} \mathbf{U}^{T} \mathbf{M U} & =\left[\begin{array}{llll}1 & & & \\ & 1 & & \\ & & \ldots & \\ & & & 1\end{array}\right] \\ \mathbf{U}^{T} \mathbf{K U} & =\left[\begin{array}{llll}\omega_{01}^{2} & & & \\ & \omega_{02}^{2} & & \\ & & \ldots & \\ & & & \omega_{0 n}^{2}\end{array}\right]=\mathbf{\Omega}\end{aligned}$

The Rayleigh damping matrix $\mathbf{D}_{\mathbf{p}}$ can be diagonalized applying $(3.6 \mathrm{a}, \mathrm{b})$ to $(3.1 \mathrm{a})$ :

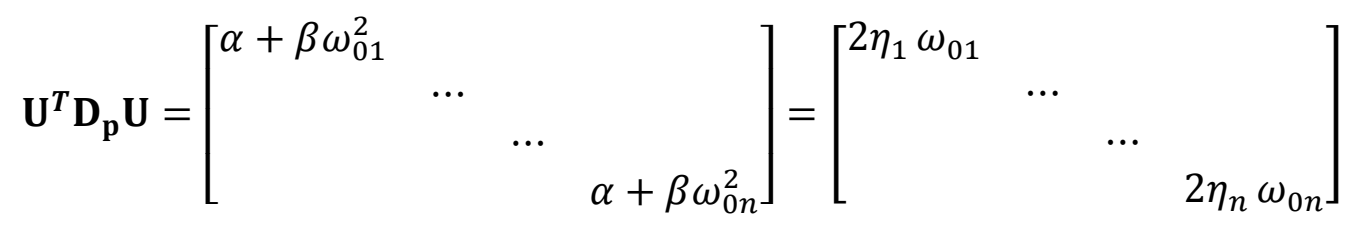

The general form of the $\mathbf{D}_{\mathbf{p}}$ matrix (3.1b) can also be transformed in diagonal form by use of the real modal matrix $\mathbf{U}$, as shown in [7] p. 105.

The terms in the main diagonal of the right side of (3.7) are set to be equal to the modal damping term $2 \eta \omega_{0}$ of the equation of motion for SDOFS (2.1). The two unknown parameter $\alpha$ and $\beta$ can be calculated by solving a system of two equations $\alpha+\beta \omega_{0 j}^{2}=2 \eta_{j} \omega_{0 j}$, $(j=1,2)$, using the first two lowest free frequencies $\omega_{0 j}$ and by arbitrary choose of two appropriate damping ratios $\eta_{j}$ :

$$
\begin{aligned}
\alpha & =\frac{2 \omega_{01} \omega_{02}\left(\omega_{01} \eta_{2}-\omega_{02} \eta_{1}\right)}{\omega_{01}^{2}-\omega_{02}^{2}} \\
\beta & =\frac{2\left(\omega_{01} \eta_{1}-\omega_{02} \eta_{2}\right)}{\omega_{01}^{2}-\omega_{02}^{2}}
\end{aligned}
$$

Typically for the viscous damping is evidently the frequency related damping parameters. Below the parameter $\alpha$ and $\beta$ are set to be known. In the general case (3.1b) the unknown coefficients $a_{k}$ are to be determined by solving a system of $\mathrm{n}$ linear equation, see [7] for details.

We consider now the proportionally damped system (1.1) with $\mathbf{D}=\mathbf{D}_{\mathbf{p}}$. Assuming the solution (1.2), the associated quadratic eigenvalue problem (1.3) gives

$$
\lambda_{j}^{2} \mathbf{M} \boldsymbol{\Phi}_{j}+\lambda_{j} \underbrace{(\alpha \mathbf{M}+\beta \mathbf{K})}_{\mathbf{D}_{\mathbf{p}}} \boldsymbol{\Phi}_{j}+\mathbf{K} \boldsymbol{\Phi}_{j}=\mathbf{0}
$$

The mass normalized eigenvectors $\boldsymbol{\Phi}_{j}$ in Eq. (3.9) are generally complex conjugate, see Eq. (2.13) - (2.15). But the eigenvalue problem (3.9) possess also "classical" real eigenmodes, identical to the eigenmodes $\mathbf{U}_{j}$, belonging to the eigenvalue problem without damping, see Eq. (3.2) - (3.5).

Using the $\mathbf{U}_{j}$ eigenmodes instead of $\boldsymbol{\Phi}_{j}$, the eigenvalue problem (3.9) can be transformed, with regard to (3.6), (3.7), to 
$\{\lambda_{j}^{2} \mathbf{M}+\lambda_{j} \underbrace{\left(\alpha+\beta \omega_{0 j}^{2}\right) \mathbf{M}}_{\mathbf{D}_{\mathbf{p}}}+\underbrace{\omega_{0 j}^{2} \mathbf{M}}_{\mathbf{K}}\} \mathbf{U}_{j}=\mathbf{0}$

The corresponding complex eigenvalue $\lambda_{j}$ is then computed from $\lambda_{j}^{2}+\lambda_{j}\left(\alpha+\beta \omega_{0 j}^{2}\right)+\omega_{0 j}^{2}=0$

$\rightarrow \lambda_{j 1,2}=-\frac{1}{2} \underbrace{\left(\alpha+\beta \omega_{0 j}^{2}\right)}_{2 \eta_{j} \omega_{0 j}} \pm \sqrt{\frac{1}{4}\left(\alpha+\beta \omega_{0 j}^{2}\right)^{2}-\omega_{0 j}^{2}}=$

$\rightarrow \quad \lambda_{j 1,2}=\underbrace{-\eta_{j} \omega_{0 j}}_{\lambda_{r}} \pm i \underbrace{\omega_{0 j} \sqrt{1-\eta_{j}^{2}}}_{\lambda_{i}}$

By comparing (3.11b) to (1.7) is evidently, that the free frequency $\omega$, computed according to $(2.4),(2.5)$

$\omega=\sqrt{\lambda_{r}^{2}+\lambda_{i}^{2}}=\left\{(\eta \omega)^{2}+\left(\omega \sqrt{1-\eta^{2}}\right)^{2}\right\}^{\frac{1}{2}}$

by use of the $\mathrm{j}^{\text {th }}$ conjugate complex eigenvalues $\lambda_{j}$ of the proportional damped system, is identic to the free frequency $\omega_{0 j}$ of the corresponding system without damping. With the eigenvalue $\lambda_{j}$, Eq.(3.11b), the relationship (3.11a) proves that $\mathbf{U}_{j}$ is a eigenvector of the proportional damped system (3.9).

In the considered case the equations of motion (1.1) can be transformed in modal space Eq. (3.12), with regard to (3.6), (3.7):

$\mathbf{U}^{T} \mathbf{M U} \ddot{\mathbf{y}}+\mathbf{U}^{T} \mathbf{D}_{\mathbf{p}} \mathbf{U} \dot{\mathbf{y}}+\mathbf{U}^{T} \mathbf{K U} \mathbf{y}=\mathbf{U}^{T} \mathbf{p}(t)$,

In (3.12) the modal superposition of the original DOF is supposed by use of the "classical" modal matrix $\mathbf{U}$ of the undamped problem, see Eq.(3.4), :

$\mathbf{V}=\left[\begin{array}{llll}\mathbf{U}_{1} & \mathbf{U}_{2} & \cdots & \mathbf{U}_{n}\end{array}\right] \cdot\left[\begin{array}{c}y_{1} \\ y_{2} \\ \cdots \\ y_{n}\end{array}\right]=\mathbf{U} \cdot \mathbf{y}$

In order to transform the state space form of the equations of motion (2.12) we construct a (2n $x 2 n)$ transformation matrix $\mathbf{Y}_{\mathrm{U}}$ by the mass normalized eigenvectors $\mathbf{U}_{j}(3.4),(3.5)$ in the form

$\mathbf{Y}_{\mathbf{U}}=\left[\begin{array}{ccccccc}\mathbf{U}_{1} & \mathbf{0} & \mathbf{U}_{2} & \mathbf{0} & \cdots & \mathbf{U}_{n} & \mathbf{0} \\ \mathbf{0} & \mathbf{U}_{1} & \mathbf{0} & \mathbf{U}_{2} & \cdots & \mathbf{0} & \mathbf{U}_{n}\end{array}\right]$

Eq. (2.12) can be transformed into $n$ uncoupled real SDOFS block equations by the aid of $\mathbf{Y}_{\mathrm{U}}$, with regard to Eqs. (3.6), (3.7): 


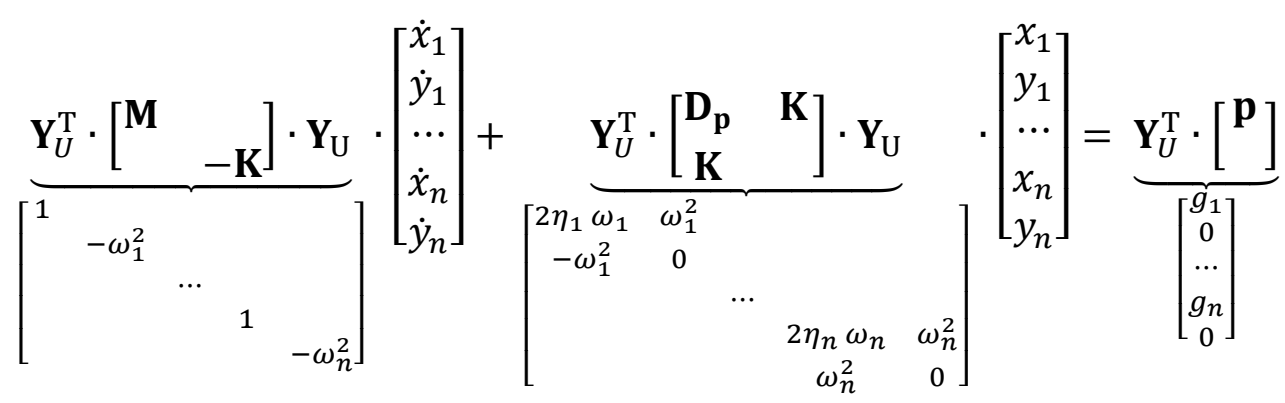

where the modal velocity is

$x_{j}=\dot{y}_{j}$

Equation (3.15) is another form of the modal decomposition (3.12). Note the difference of Eq. (3.16) from Eq.(2.30a) in the general case of non-proportional damping, see further Eq. (3.17).

We compare now the transformed equations (3.15) for the case of proportional damping with the general form (2.23) where $\mathbf{D}=\mathbf{D}_{p}$. For both of the compared SDOFS block equations to be identic, it is evidently that each "load" term $h_{j}$ from (2.23) must be equal to zero, see $(2.29 b)$ :

$h=\frac{\omega^{2}}{\sqrt{1-\eta^{2}}}\left\{Z_{1} \boldsymbol{\Phi}_{\mathbf{r}}^{\mathbf{T}}-Z_{2} \boldsymbol{\Phi}_{\mathbf{i}}^{\mathbf{T}}\right\} \mathbf{p}=0$

All terms in Eq. (3.17) exclusive of $\mathbf{p}$ belong to the considered $j^{\text {th }}$ eigenmode. Thus, with regard to $(2.11 \mathrm{a}),(2.5)$

$$
\begin{aligned}
& \left(Z_{1} \boldsymbol{\Phi}_{\mathbf{r}}^{\mathbf{T}}-Z_{2} \boldsymbol{\Phi}_{\mathbf{i}}^{\mathbf{T}}\right)=\mathbf{0} \\
& \rightarrow \quad \frac{\Phi_{i(k)}}{\Phi_{r(k)}}=\frac{Z_{1}}{Z_{2}}=\frac{\sqrt{\sqrt{1-\eta^{2}}+\left(1-\eta^{2}\right)}}{\sqrt{\sqrt{1-\eta^{2}}-\left(1-\eta^{2}\right)}}=\frac{\eta}{1-\sqrt{1-\eta^{2}}}=\text { const } .
\end{aligned}
$$

for all $\mathrm{k}^{\text {th }}$ DOF of the $\mathrm{j}^{\text {th }}$ eigenmode pair $\left(\boldsymbol{\Phi}_{\mathbf{r}} \pm i \boldsymbol{\Phi}_{\mathbf{i}}\right)$ with corresponding eigenvalue $\left(\lambda_{r} \pm i \lambda_{i}\right)$. Equation (3.18) proves the statement of a constant phase lag/lead, see Eq. (1.10), i.e. in the case of proportionally damped system each free vibration is a synchronous motion of all DOF.

\subsection{The transformation matrix $Y$}

The modal equations (3.15) demonstrate, that for the investigated case of proportional damping the modal transformation matrix $\mathbf{Y}_{\mathrm{U}}$, Eq. (3.14), must be identical to the matrix $\mathbf{Y}$, Eq. (2.23), (2.24), derived for the case $\mathbf{D}=\mathbf{D}_{p}$. By comparing the two columns of $\mathbf{Y}$, see Eq. $(2.27 \mathrm{~b}, \mathrm{c})$, to the corresponding zero-columns of $\mathbf{Y}_{\mathrm{U}}$, it follows

$$
\begin{aligned}
& \mathbf{Y}_{\mathbf{y}}^{\mathbf{W}}=\frac{1}{\sqrt{1-\eta^{2}}}\left(\omega^{2} Z_{1} \boldsymbol{\Phi}_{\mathbf{r}}-\omega^{2} Z_{2} \boldsymbol{\Phi}_{\mathbf{i}}\right)=\mathbf{0} \\
& \mathbf{Y}_{\mathbf{x}}^{\mathbf{V}}=\frac{1}{\sqrt{1-\eta^{2}}}\left(-Z_{1} \boldsymbol{\Phi}_{\mathbf{r}}+Z_{2} \boldsymbol{\Phi}_{\mathbf{i}}\right)=\mathbf{0}
\end{aligned}
$$


$\rightarrow \quad-Z_{1} \boldsymbol{\Phi}_{\mathbf{r}}+Z_{2} \boldsymbol{\Phi}_{\mathbf{i}}=\mathbf{0}$

The relationship (3.20) leads again to $\frac{\Phi_{i(k)}}{\Phi_{r(k)}}=\frac{z_{1}}{Z_{2}}=$ const. (Eq.(3.18)) for all $\mathrm{k}^{\text {th }} \mathrm{DOF}$ of the considered eigenmode.

Thus the modal transformation matrix Y, see Eq. (2.25) and (3.14), has in this case the form

$\mathbf{Y}=\left[\begin{array}{cccc}\cdots & \mathbf{Y}_{\mathbf{x}}^{(j) \mathbf{W}} & \mathbf{0} & \cdots \\ \cdots & \mathbf{0} & \mathbf{Y}_{\mathbf{y}}^{(j) \mathbf{V}} & \cdots\end{array}\right]=\left[\begin{array}{cccc}\cdots & \mathbf{U}_{j} & \mathbf{0} & \cdots \\ \cdots & \mathbf{0} & \mathbf{U}_{j} & \cdots\end{array}\right]$

\section{NUMERICAL EXAMPLE}

\subsection{Structural system, stiffness and geometry data}
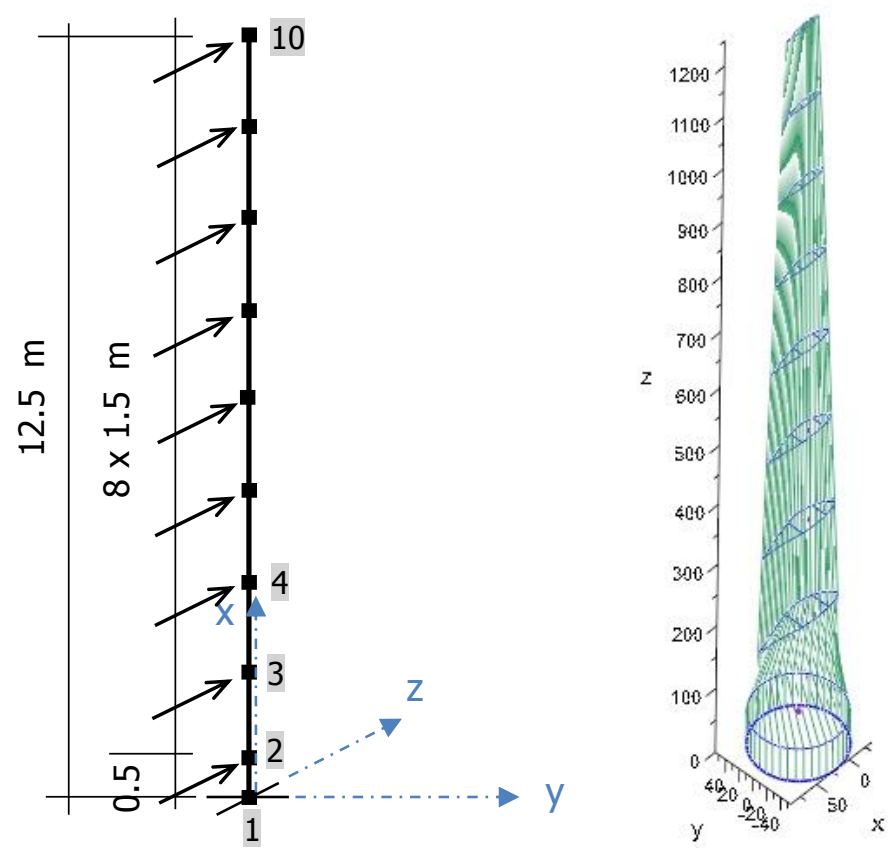

Fig. 1 Rotor blade beam model subjected to wind loads

The stiffness data of the blade cross sections have been calculated in [14]. The generic aerodynamic blade geometry has been derived from real blade data. Below are given for instance the stiffness data, referred to the origin of the coordinate system of the cross section, at the distance of $2.0 \mathrm{~m}$ from the blade root - see Fig. 2:

Center of mass F $(0.124,-0.0119)[\mathrm{m}]$

$\mathrm{EA}=947410000.0 \quad[\mathrm{~N}]$ (axial stiffness)

$\mathrm{EA}_{\mathrm{y}}=86101990.0[\mathrm{Nm}]$

$\mathrm{EA}_{\mathrm{yy}}=48655550.0\left[\mathrm{Nm}^{2}\right]$

$\mathrm{EA}_{\mathrm{yz}}=281046.0\left[\mathrm{Nm}^{2}\right]$ distributed mass

$73.835[\mathrm{~kg} / \mathrm{m}]$

$$
\begin{aligned}
& \mathrm{EA}_{\mathrm{z}}=-4408355.0[\mathrm{Nm}] \\
& \mathrm{EA}_{\mathrm{zz}}=16441220.0\left[\mathrm{Nm}^{2}\right] \\
& \mathrm{GI}_{\mathrm{T}}=6500099.0\left[\mathrm{Nm}^{2}\right] \text { (torsional stiffness) }
\end{aligned}
$$


The finite element solution is based on the numerical integration of the system of differential equations for the Bernoulli-beam. The reference axis of the beam model coincides with the centre of the circular-section at the root - it is the real rotational axis of the rotor blade. Thereby the differential equations and all cross section stiffness data are refered to this axis, accounting for the eccentric mass application.

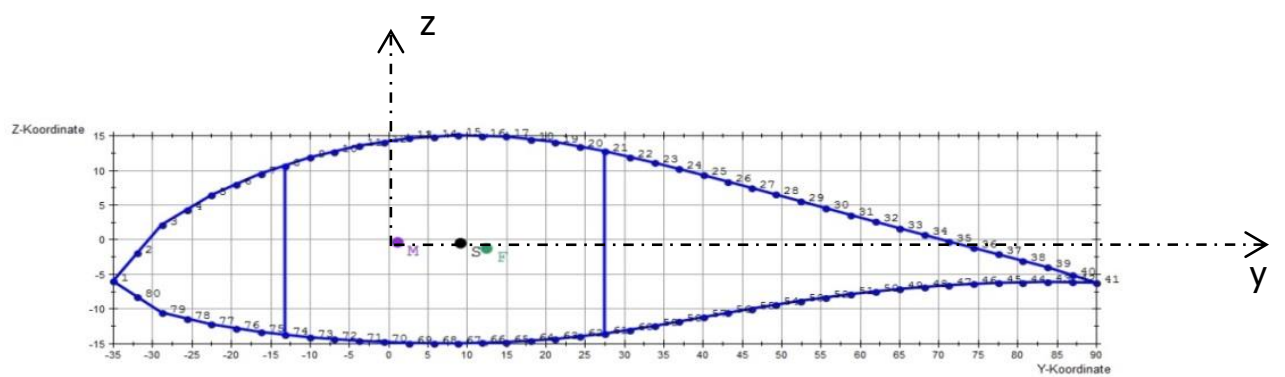

Fig. 2 Rotor blade sections at $2.0 \mathrm{~m}$ - thin wall cross section model

\subsection{Wind loads}

The wind loads are calculated according to the formula for the aerodynamic lift force per unit length of an aerofoil, see [13] p.59:

$L=\frac{1}{2} \rho \cdot c(r) \cdot W^{2} \cdot C_{L}$

where: $\quad W \quad$ : air velocity relative to the aerofoil

$\rho \quad:$ air density $=1.225\left[\mathrm{~kg} / \mathrm{m}^{3}\right]$

$c(r) \quad$ : chord of the aerofoil

$C_{L} \quad:$ lift coefficient $C_{L}=2 \pi \alpha=2 \pi\left(\frac{\pi}{180} 6.0\right)=0.658$,

the flow angle $\alpha$ is assumed to be 6.0 [deg]

The air velocity $W$ is the vector sum of the rotational speed $\Omega$ (with assumed $60 \mathrm{rpm}$ ) and the wind speed $u$, incident on the aerofoil in accordance with the Betz-theory:

$W=\sqrt{(\Omega r)^{2}+\left(\frac{2}{3} u\right)^{2}} \quad$ where $\Omega=\left(\frac{60}{30} \pi\right)$ in $[\mathrm{rad} / \mathrm{s}]$

The wind speed function is assumed to be

$u(t)=16.0+8.0 \sin (2 \pi f t), \quad$ where $\quad f=1[\mathrm{~Hz}]$

The resulting wind thrust loads per unit length along the $\mathrm{x}$-axis of the rotor blade are given below. In the structural model the wind thrust loads are acting as summarized nodal forces. 
$\mathrm{r} \quad \mathrm{c} \quad$ wind thrust $\mathrm{F}(\mathrm{t})$

[m] $[\mathrm{m}]$

$[\mathrm{N} / \mathrm{m}]$

$\left(\begin{array}{ccc}0 & 1.1 & 0 \\ 0.5 & 1.1 & 0.440881(5.33333 \sin (6.28319 t)+10.6667)^{2}+4.35132 \\ 2.0 & 1.25 & 0.501001(5.33333 \sin (6.28319 t)+10.6667)^{2}+79.115 \\ 3.5 & 1.15 & 0.460921(5.33333 \sin (6.28319 t)+10.6667)^{2}+222.906 \\ 5.0 & 1.05 & 0.420841(5.33333 \sin (6.28319 t)+10.6667)^{2}+415.354 \\ 6.5 & 0.95 & 0.380761(5.33333 \sin (6.28319 t)+10.6667)^{2}+635.095 \\ 8.0 & 0.85 & 0.340681(5.33333 \sin (6.28319 t)+10.6667)^{2}+860.771 \\ 9.5 & 0.75 & 0.300601(5.33333 \sin (6.28319 t)+10.6667)^{2}+1071.02 \\ 11.0 & 0.65 & 0.260521(5.33333 \sin (6.28319 t)+10.6667)^{2}+1244.48 \\ 12.5 & 0.55 & 0.220441(5.33333 \sin (6.28319 t)+10.6667)^{2}+1359.79\end{array}\right)$

The wind thrust functions $F(t)$ are acting on the rotor blade as shown in Fig. 3 for 10 sec.

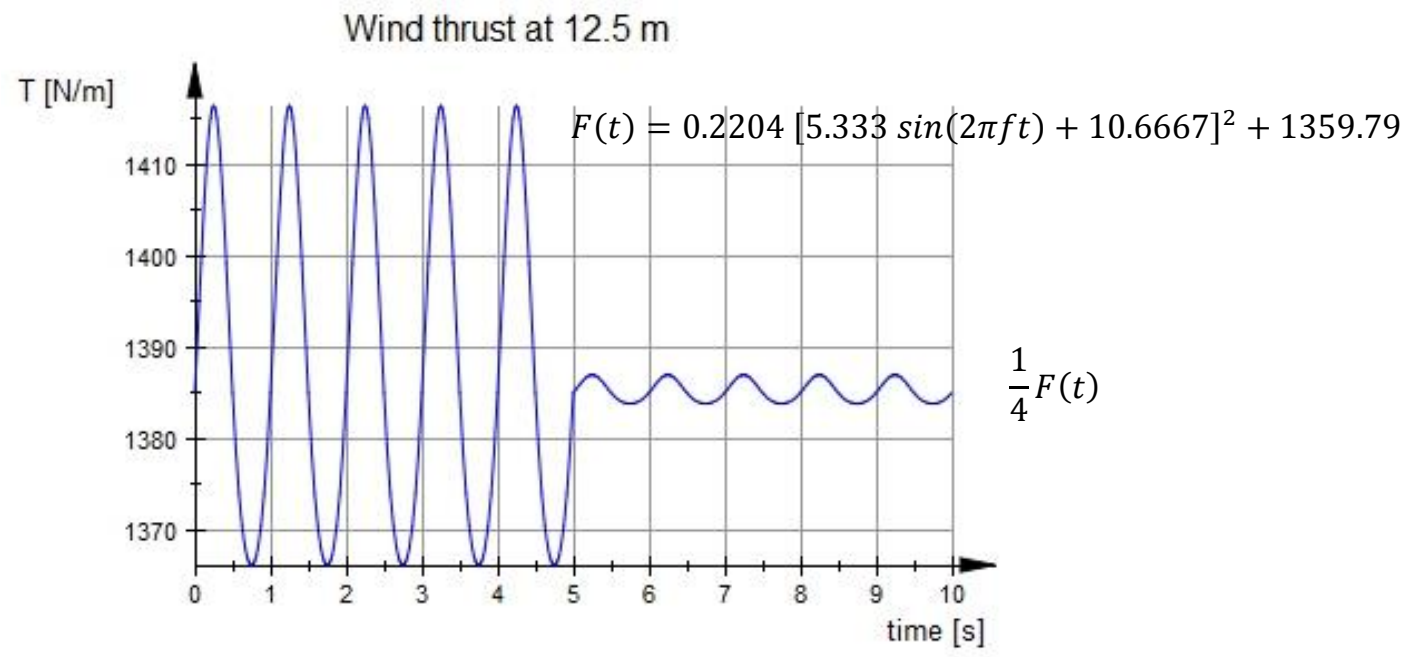

Fig. 3 Wind thrust function at $12.5 \mathrm{~m}$

\subsection{Relationships and data for the damping approach}

Starting point of the computation are the equations of motion

$\left[\begin{array}{cc}\mathbf{M} & \mathbf{0} \\ \mathbf{0} & -\mathbf{K}\end{array}\right]\left[\begin{array}{c}\dot{\mathbf{W}} \\ \dot{\mathbf{V}}\end{array}\right]+\left[\begin{array}{cc}\mathbf{D} & \mathbf{K} \\ \mathbf{K} & \mathbf{0}\end{array}\right]\left[\begin{array}{l}\mathbf{W} \\ \mathbf{V}\end{array}\right]=\left[\begin{array}{c}\mathbf{P}(t) \\ \mathbf{0}\end{array}\right]$

where $\mathbf{P}(t)$ is the nodal force vector, representing the wind thrust according to Sec. 4.2.

The system equations (4.4) will be solved applying the proposed modal analysis method in Sec. 2 for two cases: non-proportional and proportional damping.

The lowest four free-vibration frequencies and associated periods for the undamped system are calculated to 


$$
\begin{array}{cl}
f_{1}=2.643\left[s^{-1}\right] & T_{1}=0.378[s] \\
f_{2}=4.622\left[s^{-1}\right] & T_{2}=0.216[s] \\
f_{3}=7.942\left[s^{-1}\right] & T_{3}=0.126[s] \\
f_{4}=16.650\left[s^{-1}\right] & T_{4}=0.060[s]
\end{array}
$$

Assuming stiffness proportional damping in accordance with Eq.(3.1), the damping system matrix is

$\mathbf{D}_{\mathbf{p}}=\beta \mathbf{K}$

With an assumed damping ratio $\eta=0.008$, see [13] p.249, for the first natural period $T_{1}$, we obtain with regard to Eq.(3.8a,b)

$\beta=\frac{2 \eta}{\omega_{1}}=\frac{\eta T_{1}}{\pi}=0.000964[s]$

The non-proportional symmetric damping matrix $\mathbf{D}_{\mathbf{n p}}$ is build adding to the $\mathbf{D}_{\mathbf{p}}$-matrix a new matrix $\mathbf{D}_{\mathbf{a}}$, which represents the aerodynamic damping. The formulation is based on a simple expression for the aerodynamic damping coefficient per unit length $c(r)$, given in [13], p. 247:

$c(r)=\frac{1}{2} \rho \cdot \Omega r \cdot c(r) \cdot \frac{d C_{L}}{d \alpha} \quad\left[\frac{k g}{s} \frac{1}{m}\right], \quad$ where $\quad \frac{d C_{L}}{d \alpha}=2 \pi$

With Eq. (4.1), (4.2), the corresponding damping coefficients along the $\mathrm{x}$-axis of the rotor blade are calculated to

$$
\begin{aligned}
& \begin{array}{lll}
\mathrm{r} & \mathrm{c} & c(r)
\end{array} \\
& \text { [m] [m] [kg/s.m] } \\
& \left(\begin{array}{ccc}
0 & 1.1 & 0 \\
0.5 & 1.1 & 13.2993 \\
2.0 & 1.25 & 60.4513 \\
3.5 & 1.15 & 97.3266 \\
5.0 & 1.05 & 126.948 \\
6.5 & 0.95 & 149.315 \\
8.0 & 0.85 & 164.428 \\
9.5 & 0.75 & 172.286 \\
11.0 & 0.65 & 172.891 \\
12.5 & 0.55 & 166.241
\end{array}\right)
\end{aligned}
$$

The coefficients $c(r)$, which represent the aerodynamic damping, are active for vibration in z-direction of the cross-section coordinate system, see Fig. 2. The associate symmetric damping matrix for the Bernoulli-beam element is derived by analogy with the method used to derive the finite element mass matrix, see [15]. Finally the symmetric system damping matrix, $\mathbf{D}_{\mathbf{n p}}$, is assembled in a finite-element manner, including structural (proportional) and aerodynamic damping:

$$
\mathbf{D}_{\mathrm{np}}=\mathbf{D}_{\mathbf{p}}+\mathbf{D}_{\mathbf{a}}
$$

\subsection{Non-proportional damped system}

We use here the matrix $\mathbf{D}_{\mathbf{n p}}$ - Eq.(4.9). The vector of the first ten complex conjugate eigenvalue pairs of the matrix $\mathbf{A}=\mathbf{M}_{\mathbf{G}}^{-1} \cdot \mathbf{K}_{\mathbf{G}}$, see Eq.(2.13), is 


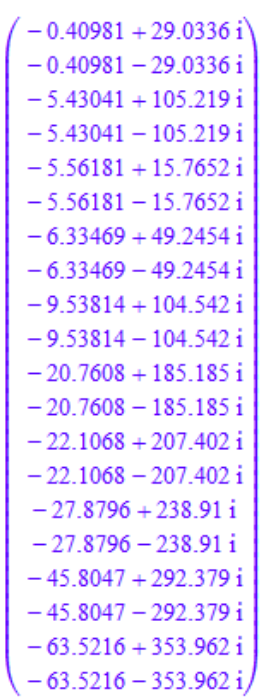

The number of modes considered in the modal transformation is limited to the first four eigenvector pairs - this are in ascending order the \#3, 1, 4, 5 of the vector in (4.10). The structural system has in Fig. 1 has 54 DOF. The corresponding $(108 \times 8)$ modal matrix $\boldsymbol{\Phi}_{\mathbf{G}}$ with mass normalized eigenvectors - Eq.(2.18), is computed to (only the first ten rows are printed)

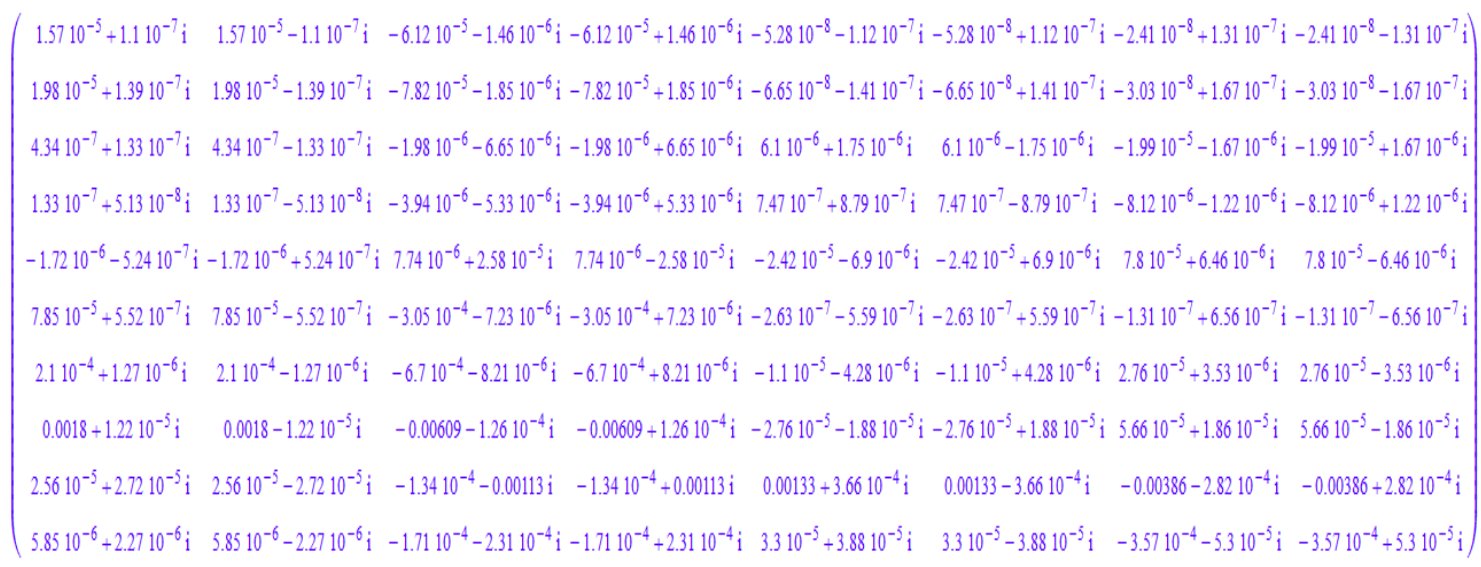

The matrix $\Psi^{-1}$ is now calculated in the case of four involved eigenmodes according to Eq. (2.24):

$\boldsymbol{\Psi}^{-1}=\left[\begin{array}{llll}\left(\varphi^{(1)}\right)^{-1} & & & \\ & \left(\varphi^{(2)}\right)^{-1} & \\ & & \left(\varphi^{(3)}\right)^{-1} & \\ & & & \left(\varphi^{(4)}\right)^{-1}\end{array}\right]=$ 


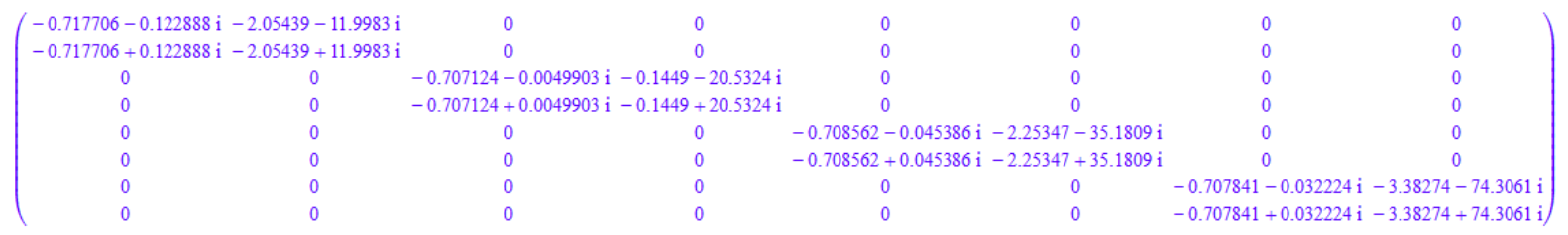

(4.12)

Finally the (108x8) real transformation matrix $\mathbf{Y}$ is computed according to (2.24) - here only the first ten rows:

$$
\mathbf{Y}=\left(\begin{array}{cccccccc}
0 & -0.000002 & -0.000022 & 0 & 0 & 0.000009 & 0 & -0.001258 \\
0 & -0.000003 & -0.000028 & 0 & 0 & 0.000012 & 0 & -0.001609 \\
-0.000008 & 0.000017 & -0.000001 & 0.000005 & 0.000028 & -0.000027 & -0.000067 & 0.000011 \\
-0.000001 & 0.000018 & 0 & 0.000002 & 0.000011 & -0.000049 & -0.000053 & 0.000259 \\
0.000033 & -0.000066 & 0.000002 & -0.000021 & -0.00011 & 0.000103 & 0.000259 & -0.000027 \\
0 & -0.000012 & -0.000111 & 0 & 0 & 0.000047 & 0.000001 & -0.00628 \\
0.000015 & -0.000058 & -0.000297 & -0.000009 & -0.000039 & 0.000124 & 0.00008 & -0.013754 \\
0.000035 & -0.000337 & -0.00255 & -0.00002 & -0.000079 & 0.001052 & 0.000202 & -0.125274 \\
-0.001819 & 0.003317 & -0.000036 & 0.001111 & 0.005438 & -0.002459 & -0.011388 & -0.001937 \\
-0.000038 & 0.000796 & -0.000008 & 0.000091 & 0.000501 & -0.002123 & -0.002276 & 0.011014
\end{array}\right)
$$

After the modal transformation in regard to (2.23) the time-dependent "load" vector (here for the time $0 . .5 \mathrm{sec}$ ) is calculated to be, see also Fig. 3,

$$
\left[\begin{array}{c}
g_{1}(t) \\
h_{1}(t) \\
\cdots \\
g_{3}(t) \\
h_{3}(t)
\end{array}\right]=\mathbf{Y}^{\mathrm{T}} \cdot[\mathbf{p}]=\left(\begin{array}{c}
-0.1878(5.333 \sin (6.283 t)+10.67)^{2}-690.3 \\
0.02234(5.333 \sin (6.283 t)+10.67)^{2}-165.7 \\
-0.003661(5.333 \sin (6.283 t)+10.67)^{2}-14.86 \\
0.07117(5.333 \sin (6.283 t)+10.67)^{2}+227.9 \\
0.04344(5.333 \sin (6.283 t)+10.67)^{2}-212.1 \\
1.025(5.333 \sin (6.283 t)+10.67)^{2}+3882.0 \\
-0.06672(5.333 \sin (6.283 t)+10.67)^{2}-114.6 \\
1871.0-0.01053(5.333 \sin (6.283 t)+10.67)^{2}
\end{array}\right)
$$

The resultant four uncoupled SDOFS block equations from type of Eq. (2.23), prepared in the form $(2.30 \mathrm{a}, \mathrm{b})$, are solved by step-by-step integration:

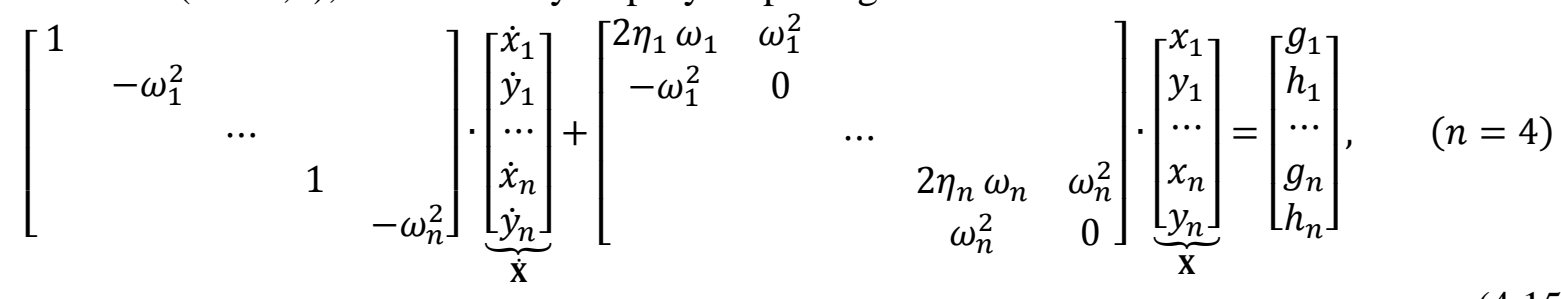

where $\quad\left[\omega_{i}\right]=\left(\begin{array}{llll}16.7175 & 29.0365 & 49.6511 & 104.976\end{array}\right)$

$$
\left[\eta_{i}\right]=\left(\begin{array}{llll}
0.332693 & 0.0141136 & 0.127584 & 0.0908603
\end{array}\right)
$$


The effect of the implied additional aerodynamic damping results evidently in the large damping ratio $\eta_{i}=0.33269$ for the first free vibration.

The vibration-response has been determined in the time $0 \ldots 10 \mathrm{~s}$, the time step length for the applied Newmark integration method is $0.005 \mathrm{~s}$.

The time response of the modal coordinates $y_{j}(t),(j=1, \ldots 4)$, are shown in the following figure 4 for the time $0 \ldots .5 \mathrm{sec}$ :
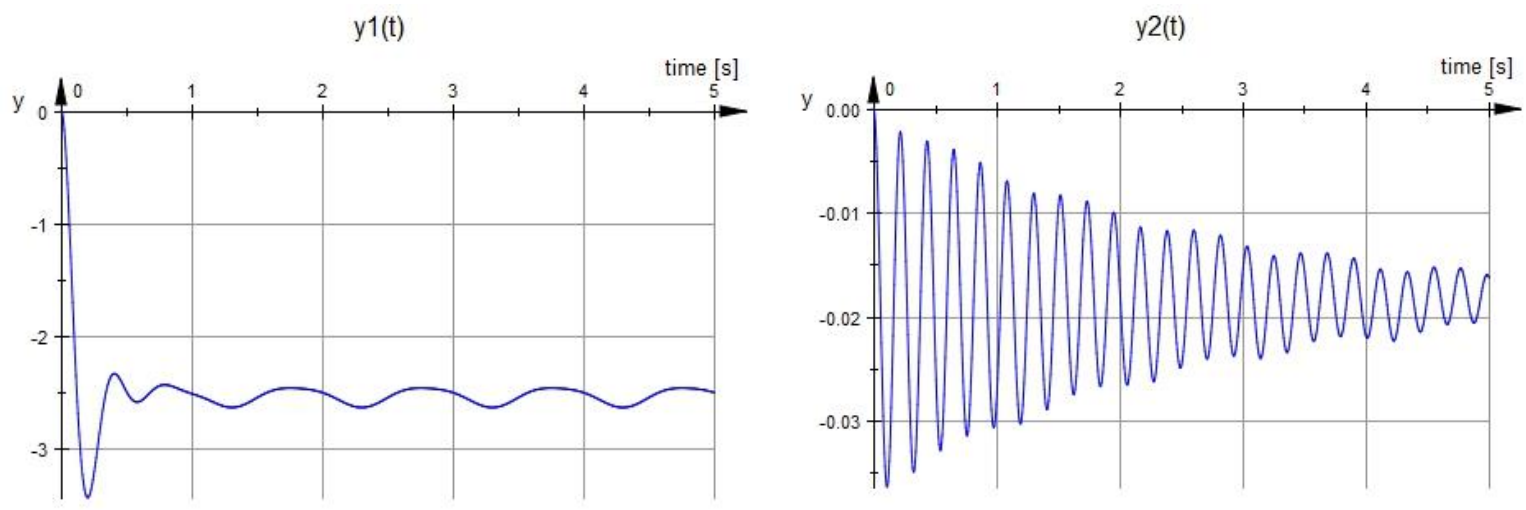

$\mathrm{y} 3(\mathrm{t})$
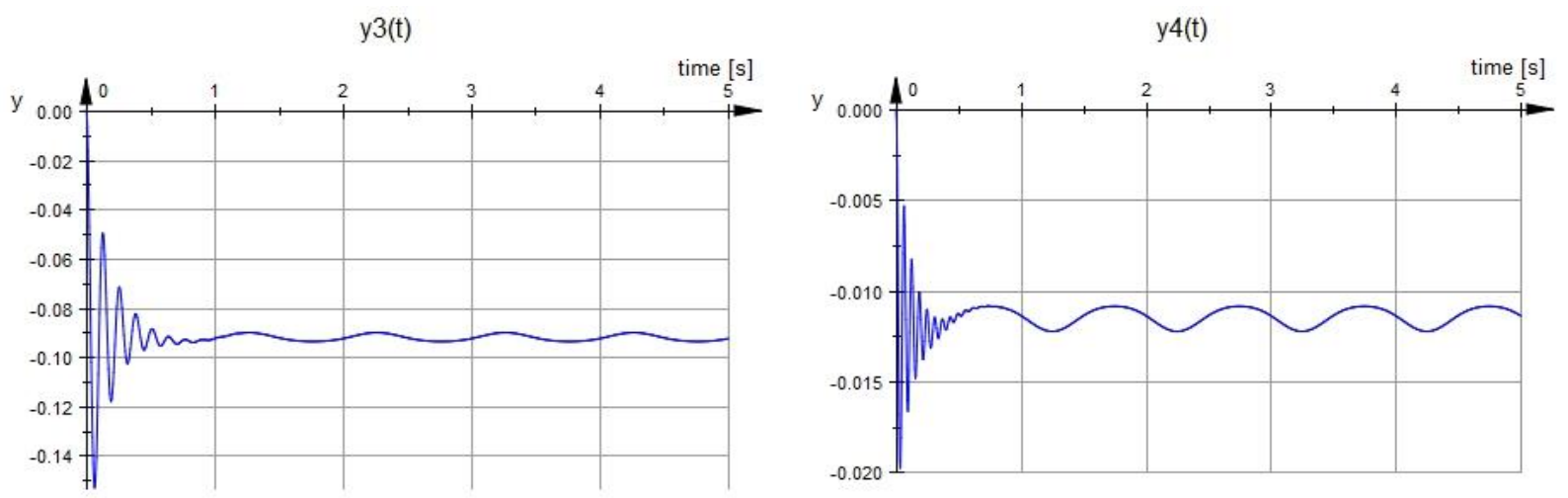

Fig. 4 Time response of the modal coordinates $y_{j}(t)$ for the case "non-proportional damping"

By a back transformation according to Eq. (2.24) the total response $\mathbf{V}(t)$ is obtained - see Figs. 5a-c:

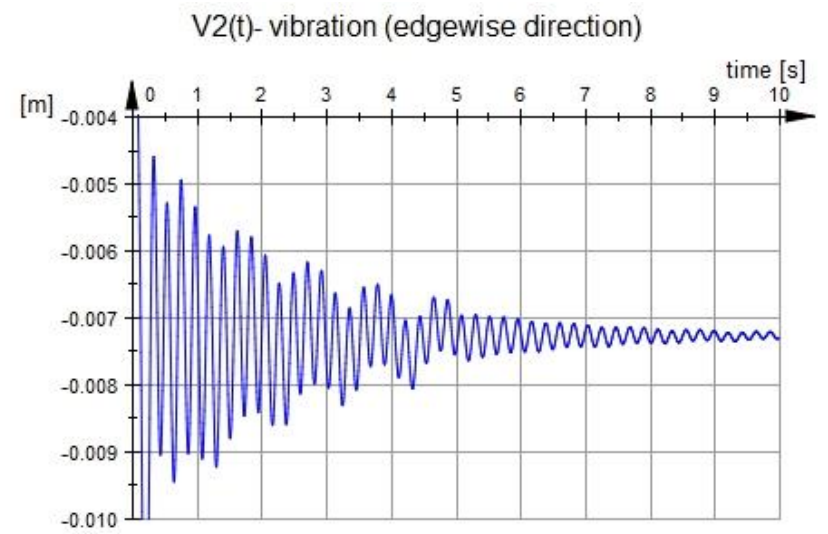

Fig.5a Total vibration $u_{2}(t)[m]$ at the rotor blade tip (y-direction at node \#10) 


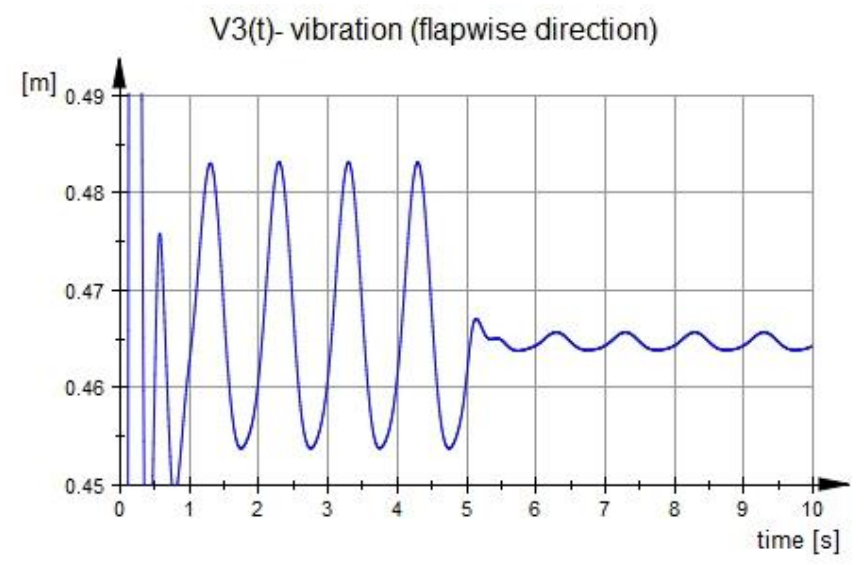

Fig.5b Total vibration $u_{3}(t)[m]$ at the rotor blade tip (z-direction at node \#10)

Phi2(t)- flapwise bending

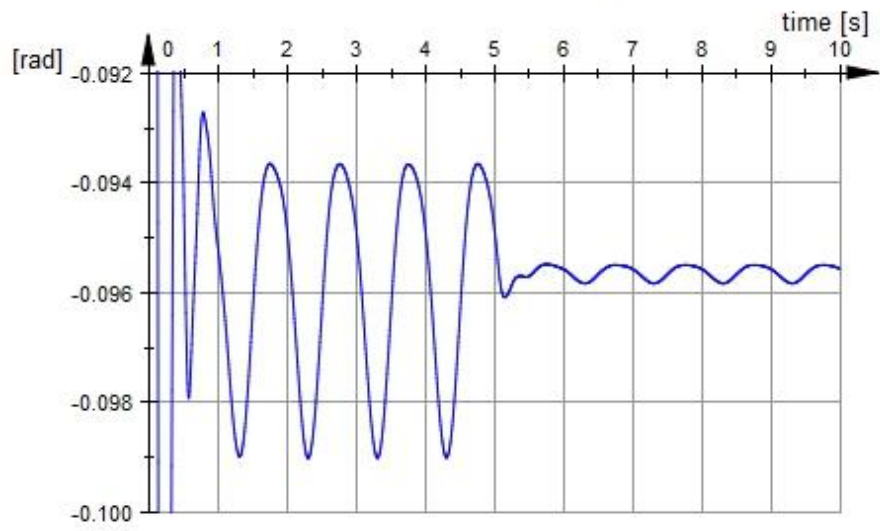

Fig.5c Total rotation $\varphi_{2}(t)[\mathrm{rad}]$ at the rotor blade tip (y-axis at node \#10)

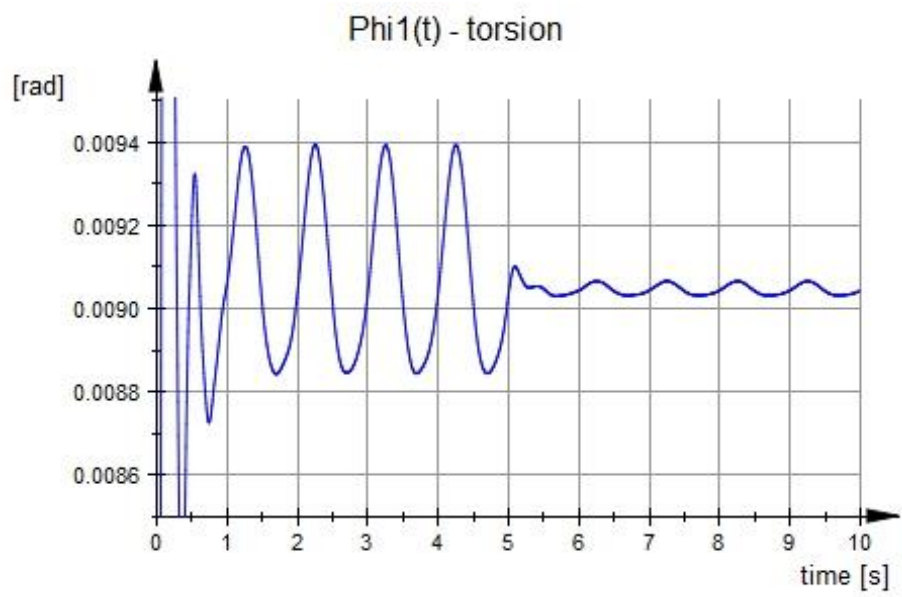

Fig.5d Total torsional rotation $\varphi_{1}(t)[\mathrm{rad}]$ at the rotor blade tip (x-axis at node \#10) 
The vibration responses, computed by direct step-by-step integration of the equations (4.4), are practically identical to those in Fig. 5a-d.

\subsection{Proportional damped system}

In this case we use the derived symmetric damping matrix $\mathbf{D}_{\mathbf{p}}-$ Eq.(4.6), (4.7). The first ten lowest complex conjugate eigenvalue pairs, resulting from Eq. (2.13), are now:

$$
\left(\begin{array}{c}
-0.132832+16.6035 \mathrm{i} \\
-0.132832-16.6035 \mathrm{i} \\
-0.406268+29.0352 \mathrm{i} \\
-0.406268-29.0352 \mathrm{i} \\
-1.19966+49.8844 \mathrm{i} \\
-1.19966-49.8844 \mathrm{i} \\
-5.27314+104.483 \mathrm{i} \\
-5.27314-104.483 \mathrm{i} \\
-5.39463+105.676 \mathrm{i} \\
-5.39463-105.676 \mathrm{i} \\
-16.7361+185.622 \mathrm{i} \\
-16.7361-185.622 \mathrm{i} \\
-20.9751+207.591 \mathrm{i} \\
-20.9751-207.591 \mathrm{i} \\
-27.8753+238.91 \mathrm{i} \\
-27.8753-238.91 \mathrm{i} \\
-42.2056+292.945 \mathrm{i} \\
-42.2056-292.945 \mathrm{i} \\
-62.3277+354.226 \mathrm{i} \\
-62.3277-354.226 \mathrm{i}
\end{array}\right)
$$

The corresponding (108x8) $\boldsymbol{\Phi}_{\mathbf{G}}$ modal matrix - Eq. (2.18), comprises the first four mass normalized complex conjugate eigenvector pairs. In order to verify the derived relationship $\frac{\Phi_{i(k)}}{\Phi_{r(k)}}=\frac{\eta}{1-\sqrt{1-\eta^{2}}}=$ const. , see (3.14), we compute this ratio for all components of the involved $\left(\boldsymbol{\Phi}_{\mathbf{r}} \pm i \boldsymbol{\Phi}_{\mathbf{i}}\right)^{(j)}(j=1, \ldots 4)$ eigenvectors (for instance the first ten rows only):

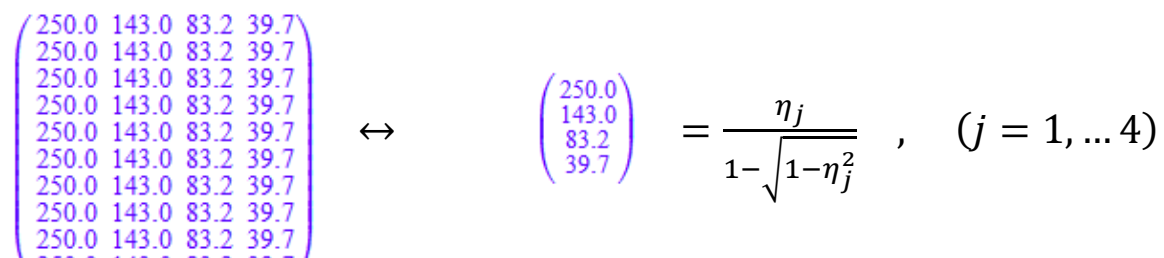

The corresponding damping ratios $\eta_{j}$, see Eq. (4.21b), are computed in accordance with Eq. (2.5).

The next step is the computation of the matrix $\Psi^{-1}$, Eq. (2.24). The (108x8) real transformation matrix $\mathbf{Y}$, computed in regard with Eq. (2.24), (2.27), has now the form of (3.21):

$$
\mathbf{Y}=\left(\begin{array}{cccccccc}
0 & 0 & -0.000022 & 0 & 0 & 0 & 0.000053 & 0 \\
0 & 0 & -0.000028 & 0 & 0 & 0 & 0.000068 & 0 \\
-0.000009 & 0 & -0.000001 & 0 & 0.000028 & 0 & -0.00005 & 0 \\
-0.000002 & 0 & 0 & 0 & 0.000011 & 0 & -0.000038 & 0 \\
0.000035 & 0 & 0.000003 & 0 & -0.00011 & 0 & 0.000195 & 0 \\
0.000001 & 0 & -0.000111 & 0 & 0 & 0 & 0.000265 & 0 \\
0.000017 & 0 & -0.000297 & 0 & -0.000039 & 0 & 0.000642 & 0 \\
0.000053 & 0 & -0.002549 & 0 & -0.000082 & 0 & 0.005423 & 0 \\
-0.001935 & 0 & -0.000051 & 0 & 0.005447 & 0 & -0.008771 & 0 \\
-0.000069 & 0 & -0.00001 & 0 & 0.000499 & 0 & -0.001632 & 0
\end{array}\right)
$$

In (4.19) are printed again only the first ten rows of $\mathbf{Y}$. 
The time-dependent "load" vector in the general modal transformed equations (2.23) is now (here for the time $0 \ldots 5 \mathrm{sec}$ ) - see also Eq. (3.15) and (4.12):

$\left[\begin{array}{l}g_{1}(t) \\ h_{1}(t) \\ \cdots \\ g_{4}(t) \\ h_{4}(t)\end{array}\right]=\left(\begin{array}{c}-0.1872(5.333 \sin (6.283 t)+10.67)^{2}-678.6 \\ 0 \\ -0.004824(5.333 \sin (6.283 t)+10.67)^{2}-18.84 \\ 0 \\ 0.03913(5.333 \sin (6.283 t)+10.67)^{2}-227.6 \\ 0 \\ -0.052(5.333 \sin (6.283 t)+10.67)^{2}-94.4 \\ 0\end{array}\right)$

Eq. (4.20) implies $x_{j}=\dot{y}_{j}$, see Eq.(3.15), (3.16), contrary to the general case Eq.(2.30a).

In the resultant four uncoupled SDOFS block equations, see (4.15), the free frequencies and the modal damping ratios are resp.

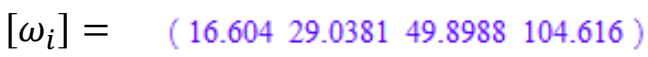

$\left[\eta_{i}\right]=\left(\begin{array}{llll}0.008 & 0.0139909 & 0.0240418 & 0.0504049\end{array}\right)$

After step-by-step integration of the four modal equations (4.15), the time series of the modal coordinates $x_{j}(t), y_{j}(t),(j=1, \ldots 4)$, are obtained - Fig. 6 :

$\mathrm{y} 1(\mathrm{t})$

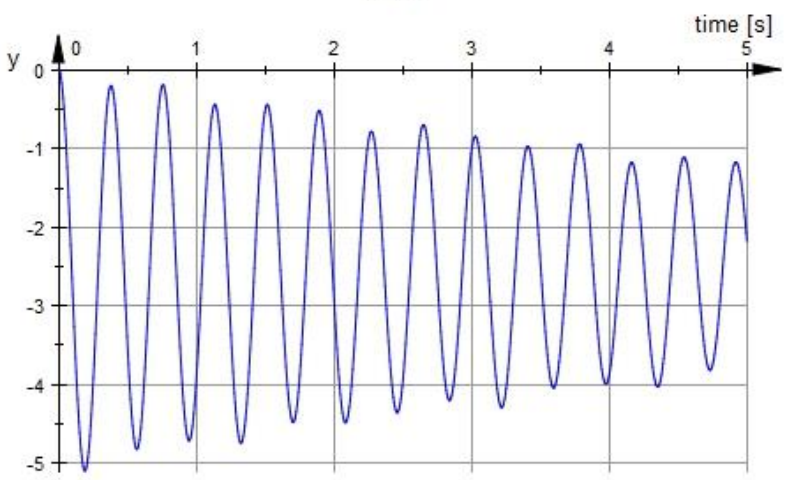

$\mathrm{y} 3(\mathrm{t})$

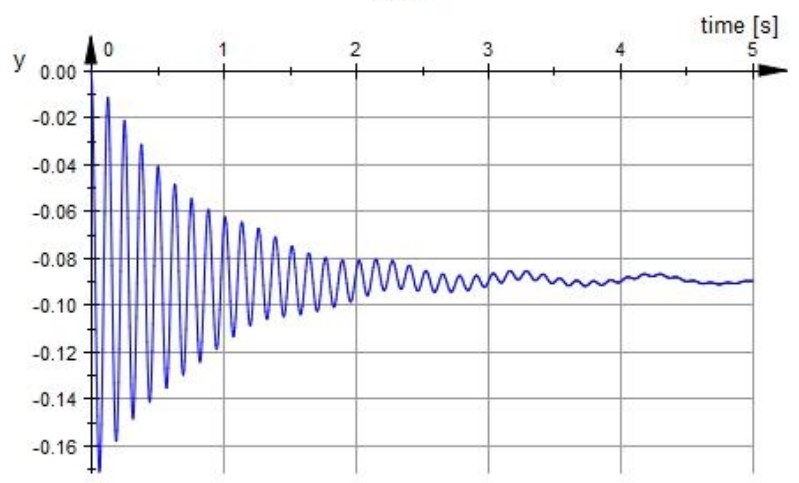

$\mathrm{y} 2(\mathrm{t})$

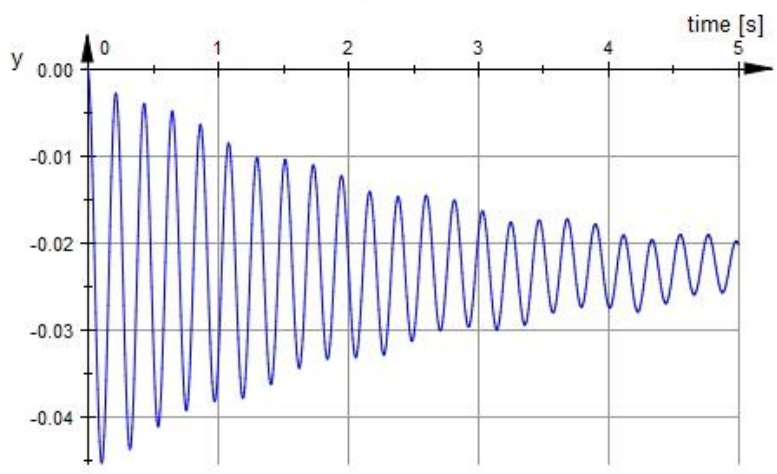

$\mathrm{y} 4(\mathrm{t})$

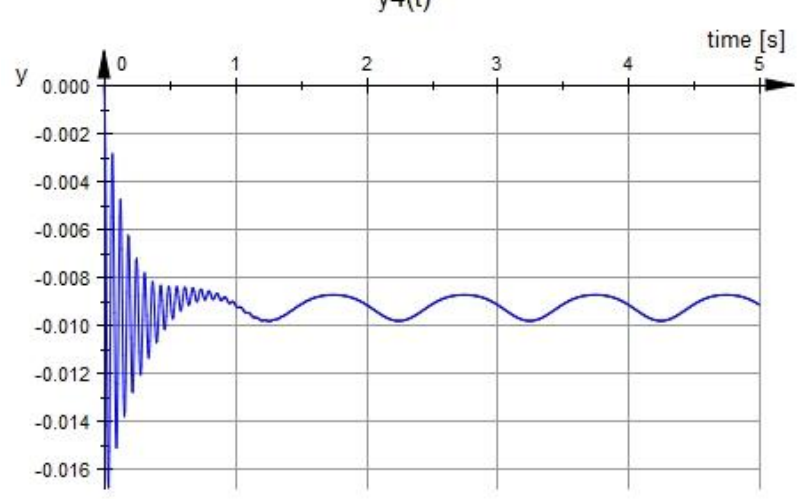

Fig. 6 Time response of the modal coordinates $y_{j}(t)$ for the case "proportional damping" 
The total responses $\mathbf{V}(t)$ are computed by a back transformation according to Eq. (2.24) - see Figs. 7a-d:

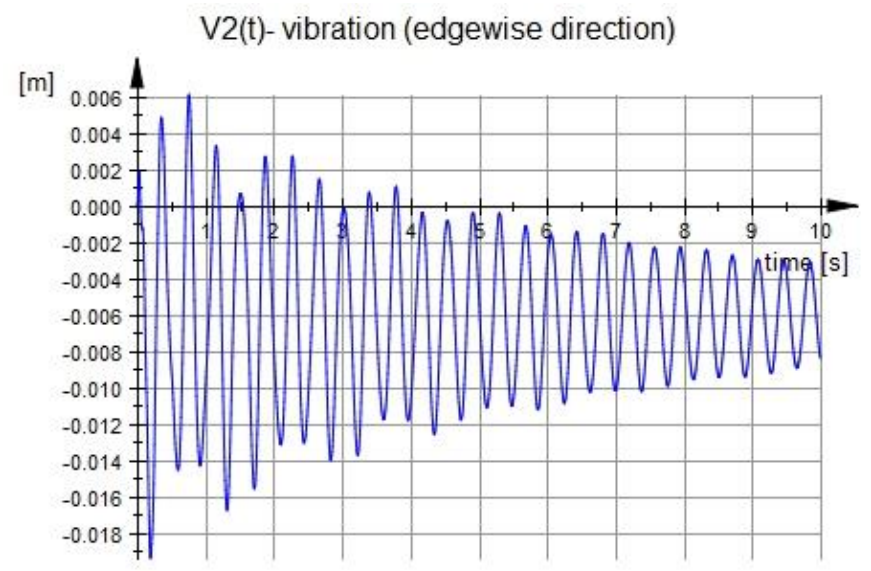

Fig 7a - Total vibration $u_{2}(t)[m]$ at the rotor blade tip (y-direction at node \#10)

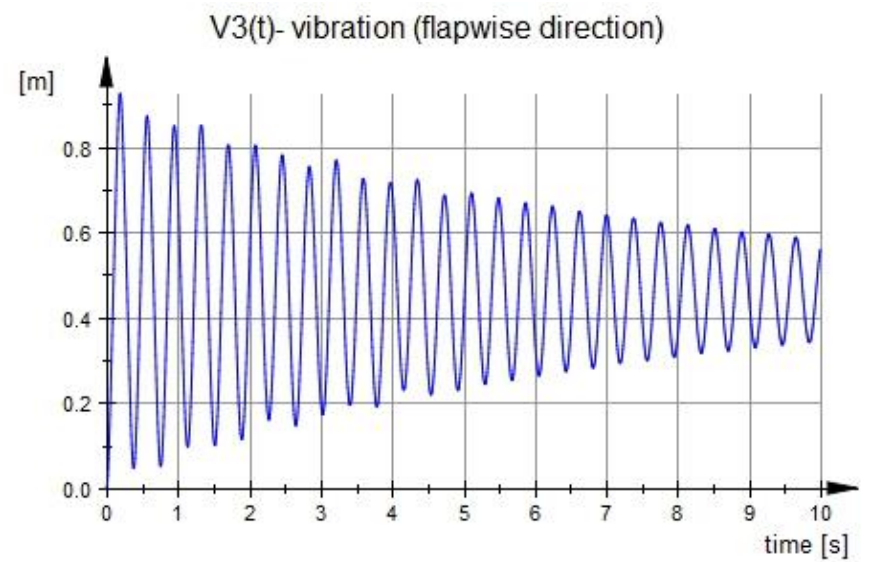

Fig $7 \mathrm{~b}$ - Total vibration $u_{3}(t)[m]$ at the rotor blade tip (z-direction at node \#10)

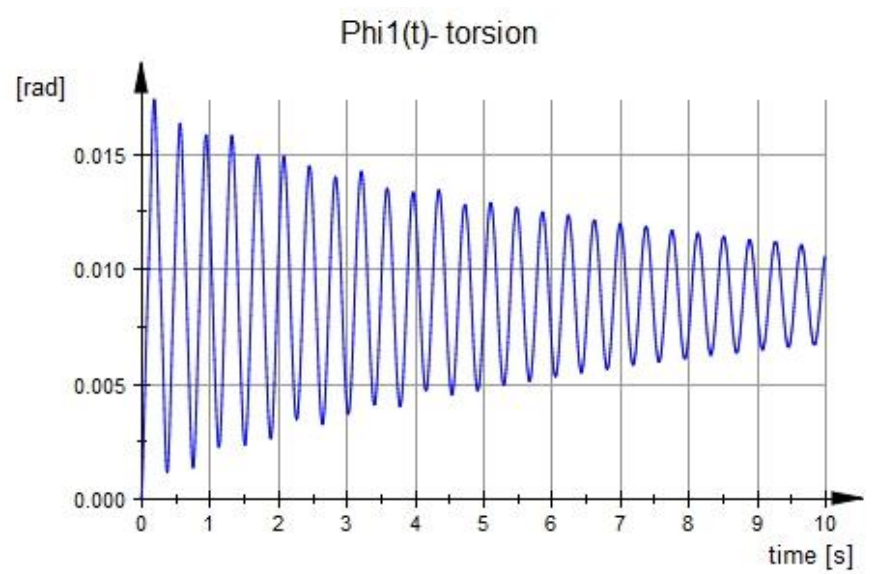

Fig 7c - Total torsion $\varphi_{1}(t)[\mathrm{rad}]$ at the rotor blade tip (x-direction at node \#10) 


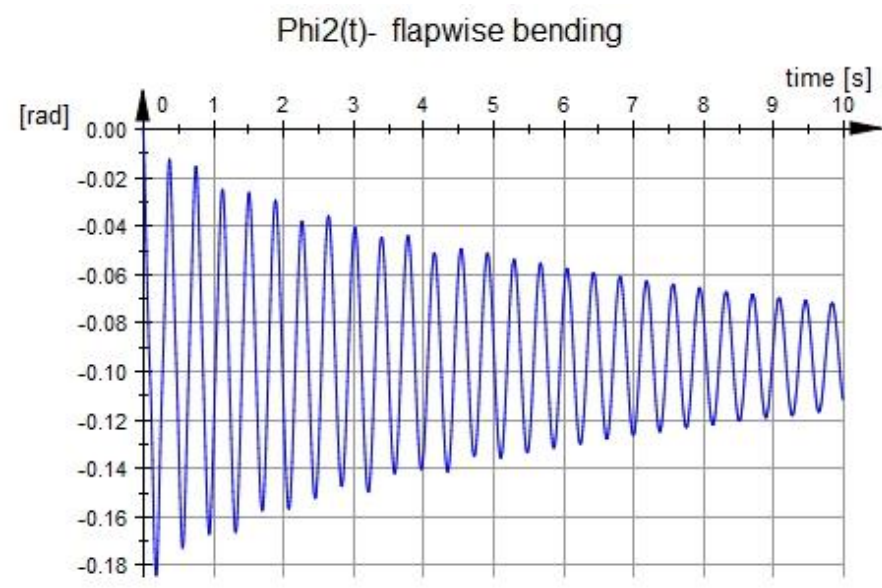

Fig $7 \mathrm{~d}$ - Total rotation $\varphi_{2}(t)[\mathrm{rad}]$ at the rotor blade tip (y-axis at node \#10)

\section{CONCLUSIONS}

- A general modal decomposition method of MDOFS with non-proportional damping is briefly presented in Sec. 2. The procedure is based on the complex eigenvalue solution of a structural model with symmetric non-proportional damping matrix. By use of the right complex conjugate eigenvector pairs, normalized relative to the general mass matrix, a new real transformation matrix Y $\mathbf{Y}$, see Eq. (2.24), (2.27), is developed analytically to perform a modal decomposition of the equations of motion in real arithmetic. The complex conjugate eigenpairs - eigenvalues and the corresponding eigenvectors - are to be computed first, at least for the lowest few modal shapes.

- The equations of motion are transformed into uncoupled SDOFS block equations. Employing only a few $(\mathrm{k})$ eigenvector pairs in the $\mathbf{Y}$-basis $(\mathrm{k}<<\mathrm{n})$ is leading - typical for a modal transformation procedure - with sufficient numerical accuracy to the total time response of all $\mathrm{n}$ DOF. The modal equations are numerically integrated and finally transformed back to the original DOF. In more details the method has been described in [3], and in [4] has been developed a similar method, based on the right and left eigenvector pairs.

- The application of the suggested method to the special case of proportional damped system is considered in details in Sec. 3. Employing a Rayleigh damping matrix, it has been shown that the modal transformation from Sec. 2 implies a ratio $\frac{\Phi_{i(k)}}{\Phi_{r(k)}}=$ const. for all $\mathrm{k}^{\text {th }}$ DOF of each considered eigenmode $\left(\boldsymbol{\Phi}_{\mathbf{r}} \pm i \boldsymbol{\Phi}_{\mathbf{i}}\right)$, i.e. the "constant phase" statement. This proves in an indirect manner that the free vibrations in the proportional damping case are synchronous. A simple formula for computing of the constant ratio has been also derived, expressing it through the associated modal damping ratio $\eta$.

- In Section 4 a numerical example - vibration of a rotor blade with 54 DOF - demonstrates the performance of the presented modal method for the two cases - nonproportional and proportional (Rayleigh) damping. In the first variant the damping matrix of the system contains a stiffness-proportional part and a simple approximated aerodynamic damping part. In the second variant the formula for the constant phase of the resonance modes is verified numerically.

- Real life applications of the proposed modal analysis method and possible numerical complications are discussed more widely in [4], [5]. The present paper studies some 
known features of proportionally damped systems - the synchronous free vibrations from a viewpoint of a new proposed modal analysis method.

\section{REFERENCES}

[1] E. Stanoev, H. Cramer. Zur Boden-Bauwerk-Interaktion auf der Basis der modalen Analyse. BKI 03/3, Universität der Bundeswehr München, München 2003, p. 135-146

[2] H. Cramer, E. Stanoev. Zur modalen Analyse bei gedämpften Mehrmassensystemen. 10. Dresdner Baustatik-Seminar: Neue Bauweisen - Trends in Statik und Dynamik, TU Dresden, Lehrstuhl für Statik, Dresden, 2006, p. 81-90

[3] H. Cramer, E. Stanoev. Ein Verfahren zur modalen Analyse gedämpfter Systeme der Strukturmechanik. Rostocker Berichte aus dem Institut für Bauingenieurwesen, Heft 19, Universität Rostock, Institut für Bauingenieurwesen, 2008, ISSN 1438-7638, p. 83-107

[4] E. Stanoev. A modified modal analysis method for damped multi-degree-of-freedomsystems in structural mechanics. Zeitschrift für angewandte Mathematik und Mechanik (ZAMM), 2013, 1 - 23 (2013), http://onlinelibrary.wiley.com/doi/10.1002/zamm.201300061/abstract

[5] E. Stanoev. A modal analysis method for structural models with non-modal damping. MS "Multibody system dynamics and modal reduction" in the frame of 11th World Congress on Computational Mechanics (WCCM XI), 5th European Conference on Computational Mechanics (ECCM V), 20-25 Juli 2014, Barcelona, ISBN: 978-84942844-7-2, Tomo IV, p. 3034-3045

[6] Peters, H. J.; Tiso, P.; Goosen, J.F.L.; van Keulen, F. Modifying resonance modes of dissipative structures using magnitude and phase information, 11th World Congress on Computational Mechanics (WCCM XI), 5th European Conference on Computational Mechanics (ECCM V), 20-25 Juli 2014, Barcelona, ISBN: 978-84-942844-7-2, Tomo II, p. 489-500

[7] Géradin, M; Rixen, D. Mechanical vibrations - theory and applications to structural dynamics, John Wiley \& Sons Ltd, 1997

[8] Meskouris, K. Baudynamik - Modelle, Methoden, Praxisbeispiele, Ernst \& Sohn, 1999

[9] Chorpa, A.K. Dynamics of Structures. Theory and Applications to Earthquake Engineering, Pearson Prentice Hall, New Jersey, 2007

[10] M.-C. Kim, L.-W. Lee. Eigenproblems for large structures with non-proportional damping. Earthquake Engng. Struct. Dyn, 28, 157-172, 1999

[11] D. C. Sorensen. Implicitly Restarted Arnoldi/Lanczos Methods for Large Scale Eigenvalue Calculations. Dep. Comp. Appl. Math., Rice University, Houston, 1995

[12] R. Lehoucq, D. C. Sorensen. Implicitly Restarted Lanczos Method. (Section 4.5,7.6) : Z. Bai, J. Demmel, J. Dongarra, A. Ruhe and H. van der Vorst, editors, Templates for the Solution of Algebraic Eigenvalue Problems: A Practical Guide, SIAM, Philadelphia, 2000, 67-80, 166-184

[13] T. Burton, T.; Jenkins, N.; Sharpe D.; Bossanyi, E. Wind Energy Handbook, John Wiley \& Sons, 2011, chapter 5.7, 5.8 
[14] Nan Li. Berechnung der Querschnittssteifigkeiten des Rotorblatts einer WEA durch ein FE-Verfahren für dünnwandige mehrzellige Profile mit Einsatz von MATLAB, Studienarbeit, University of Rostock, Chair of Wind Energy Technology, 2015

[15] E. Stanoev. Eine alternative FE-Formulierung der kinetischen Effekte beim räumlich belasteten Stab. Rostocker Berichte aus dem Institut für Bauingenieurwesen, Heft 17, Universität Rostock, Institut für Bauingenieurwesen, 2007, ISSN 1438-7638, p.143-161 